\title{
Newsvendor Bounds and Heuristics for Serial Supply Chains with Regular and Expedited Shipping
}

\author{
Sean X. Zhou, ${ }^{1}$ Xiuli Chao ${ }^{2}$ \\ ${ }^{1}$ Department of Systems Engineering and Engineering Management, The Chinese University of Hong Kong, \\ Shatin, New Territories, Hong Kong \\ ${ }^{2}$ Department of Industrial and Operations Engineering, University of Michigan, Ann Arbor, Michigan 48109-2117
}

Received 31 October 2007; revised 20 September 2009; accepted 2 October 2009

DOI 10.1002/nav.20388

Published online 19 November 2009 in Wiley InterScience (www.interscience.wiley.com).

\begin{abstract}
We study an infinite-horizon, $N$-stage, serial production/inventory system with two transportation modes between stages: regular shipping and expedited shipping. The optimal inventory policy for this system is a top-down echelon base-stock policy, which can be computed through minimizing $2 N$ nested convex functions recursively (Lawson and Porteus, Oper Res 48 (2000), 878-893). In this article, we first present some structural properties and comparative statics for the parameters of the optimal inventory policies, we then derive simple, newsvendor-type lower and upper bounds for the optimal control parameters. These results are used to develop near optimal heuristic solutions for the echelon base-stock policies. Numerical studies show that the heuristic performs well. (C) 2009 Wiley Periodicals, Inc. Naval Research Logistics 57: 71-87, 2010
\end{abstract}

Keywords: multi-echelon systems; echelon base-stock policy; bounds and heuristics; regular and expedited shipping

\section{INTRODUCTION}

Dynamic leadtime management in supply chains can hedge against market fluctuations and effectively balance inventory and customer demand. To achieve flexibility in leadtimes in a supply chain, a widely adopted strategy is the use of multiple transportation modes. Although a replenishment with shorter leadtime can better respond to customer demand, it is usually more costly. It is, therefore, important for the company to strategically determine the shipping quantities using different leadtime and cost combinations based on the inventory status to minimize its total operational costs.

In this article, we consider a periodic-review serial supply chain with $N$ stages. Each stage replenishes its inventory from its immediate upstream stage and random customer demand occurs at the most downstream stage. Excess demand in each period is backlogged. Two modes of transportation are available between any adjacent stages, regular shipping and expedited shipping, and transportation leadtimes are 1 and 0 , respectively (an extension to a more general leadtime setting is discussed in Section 5). The expedited shipping cost is higher than the regular shipping cost. The zero leadtime allows the firm to ship products from any upstream stage to

Correspondence to: S.X. Zhou (zhoux@se.cuhk.edu.hk) most downstream stage in no time, if needed, by using expedited shipping between stages. There is a linear holding cost at each stage, and a linear shortage cost at stage 1 when a backlog of customer demand occurs. The objective is to minimize the total discounted cost of the system over an infinite planning horizon.

This problem has been studied by Lawson and Porteus [13], who demonstrated that a top-down echelon base-stock policy was optimal (see also Ref. 14). A top-down echelon basestock policy is characterized by two base-stock levels for each stage, one for expedited ordering and the other for regular ordering. These optimal echelon base-stock levels can be obtained by solving $2 N$ nested single-dimensional convex optimization problems recursively. Although the algorithm itself is quite simple, computation remains a tedious process, and its complexity increases with the number of stages. This motivates us to develop simple newsvendor bounds and heuristics for the optimal policies of each stage of the multiechelon system, which not only can increase their implementability but also shed light on the effect of system control parameters.

This article makes two main contributions to the literature. First, we provide several important structural results for the optimal expedited and regular base-stock levels. These findings could advance our understanding of the system and the 
properties of the optimal policies. Second, based on these structural properties, we develop three sets of newsvendor upper bounds and three sets of newsvendor lower bounds for the optimal echelon base-stock levels for both the regular and expedited shipping modes. These bounds can be easily calculated from the system parameters. A simple heuristic is then constructed based on these bounds to compute the nearoptimal base-stock levels. Numerical studies show that the heuristic performs well.

The rest of the article is organized as follows. In Section 2 , we review the findings of previous studies in this field. In Section 3, we explain the model formulation and present some preliminary results. We provide a computational algorithm for the optimal base-stock levels and give some structural results. In Section 4, we derive several sets of lower bounds and upper bounds for the optimal echelon base-stock levels. In Section 5, we extend the results to a case with more general leadtimes. In Section 6, we develop a simple heuristic and test its effectiveness by numerical studies. We conclude the article with a few remarks in Section 7. The omitted proofs are provided in the Appendix.

Throughout the article, we use the terms "expedited order" and "expedited shipping" interchangeably. We also use the terms "increasing" and "decreasing" in a weak sense, as representing "non-decreasing" and "non-increasing," respectively. $\mathbf{1}(A)$ is the indicator function taking value 1 if $A$ is true, and 0 otherwise. For any real numbers $a$ and $b$, $a \wedge b=\min \{a, b\}, a \vee b=\max \{a, b\}$. For any realvalue, monotone function $f(\cdot)$, we use $f^{-1}(\cdot)$ to represent its inverse function. Whenever possible, we follow the notation of Lawson and Porteus [13].

\section{LITERATURE REVIEW}

The body of research related to this work can be divided into two major categories. The first analyzes control policies for single-stage and multi-stage inventory models with multiple transportation modes, and the second develops simple bounds and heuristics for the optimal control parameters. We review the various studies in these two categories separately.

The earliest study of inventory models with two delivery modes was made by Barankin [1], who studied a single-period problem. Daniel [5] was the first to consider a multi-period single-stage model with two shipping modes. The leadtimes of regular shipping and emergency shipping were 1 and 0 , respectively. Fukuda [10] extended Daniel [5] to the case where the leadtimes of the two supply modes were $L$ and $L+1$, respectively, for a general non-negative value of $L$. Whittemore and Saunders [20] considered the dual-supplier problem with leadtimes of arbitrary length, and demonstrated that the optimal control policy was very complicated and state-dependent if the difference in leadtimes was greater than 1. Because of the complexity of systems with general leadtimes, Scheller-Wolf et al. [15] and Veeraraghavan and Scheller-Wolf [19] focused on the evaluation and optimization of two classes of heuristic policies, viz., "single index" and "dual index" policies. Recently, Sheopuri et al. [17] have shown that the classical lost sales inventory problem is a special case of the dual supply modes problem. They also proposed two classes of heuristic policies and showed that one of them provided an average cost saving of $1.1 \%$ over the best "dual index" policy of Veeraraghavan and Scheller-Wolf [19]. All these studies have focused solely on single-stage inventory systems. Other related work on single-stage inventory systems with multiple transportation modes has been done by Feng et al. [8,9] and Song and Zipkin [18].

For multi-echelon models with the option of expedited shipping between stages, Lawson and Porteus [13] considered both finite-horizon and infinite-horizon serial systems with dual transportation modes. Under the assumptions that the leadtimes for regular and expedited shipping between any two adjacent stages were 1 and 0 , respectively, and that shipping costs were additive linear, they established the optimality of a top-down echelon base-stock policy. For such a policy, the control parameters of each echelon consist of two numbers, one for regular shipping and the other for expedited shipping. Muharremoglu and Tsitsiklis [14] extended the model of Lawson and Porteus [13] to a more general setting by using the unit analysis approach. Under a "supermodular" cost structure on expedited and regular shipping, they characterized the optimal policy as an extended echelon base-stock type.

When there is only one transportation mode, the system of Lawson and Porteus [13] is reduced to the classical ClarkScarf model, which has been extensively studied, notably by Clark and Scarf [4], Federgruen and Zipkin [7], and Chen and Zheng [3]. Several studies on simple bounds of cost and optimal policies for the Clark-Scarf model have been reported. Gallego and Zipkin [11] discussed the issue of stock positioning and constructed three heuristics to calculate the average cost for serial production-transportation systems. Zipkin [21] introduced a lower bound for a two-stage system by restricting the possibility of holding inventory at the upper stream stage. Dong and Lee [6] developed a lower bound for optimal policies of infinite-horizon serial systems with discounted cost criterion. For average cost criterion, Shang and Song [16] obtained simple newsvendor-type of bounds and developed simple heuristics using a different approach from that of Dong and Lee [6]. More recently, Chao and Zhou [2] have adopted another approach, constructing bounds and heuristics for serial systems that work for both discounted and average costs, and obtaining a series of bounds for optimal base-stock levels. A related study on bounds and heuristics for serial systems has been made by Gallego and Özer [12]. 
Comparing the analysis of this article to that in Chao and Zhou [2] for the Clark-Scarf model with an infinite horizon, we note the following two main differences. First, due to the existence of expedited ordering, each stage's regular order decision in each period depends on its expedited order decision of the next period, which in turn intertwines with its downstream stage's regular order decision. However, in the Clark-Scarf model, the decision in one period does not depend on that of the following period since the echelon base-stock level is myopic and stationary. Second, our system incurs two types of induced penalty cost. One occurs between adjacent stages (the cost charged from regular order of stage $i$ to stage $i+1$ due to the insufficient stock at stage $i+1$ ), and the other within each stage (the cost charged from expedited order to regular order of stage $i$ due to insufficient stock from the regular order), whereas the Clark-Scarf model features only one induced penalty cost between stages. Our analysis also shows that the expedited option between stages cannot be simply considered as an additional stage in the ClarkScarf model. These two differences make the derivation of distribution-function solutions and simple lower and upper bounds much more complicated and challenging.

\section{THE MODEL AND STRUCTURAL RESULTS}

Consider an infinite-horizon, periodic-review serial inventory system with $N$ stages, indexed by $1,2, \ldots, N$. Customer demand occurs at stage 1 . Stage 1 obtains supplies from stage 2 , stage 2 from stage 3 , and so on, and stage $N$ replenishes its inventory from an outside source (stage $N+1$ ) with an ample supply. Unsatisfied demand is fully backlogged at stage 1 . Demands in different periods are independent and identically distributed (i.i.d.) non-negative random variables. Each stage has two ordering decisions: expedited order and regular order. For each stage $i$, the leadtime $l_{i}^{\mathrm{r}}$ for regular order is 1 , and $l_{i}^{\mathrm{e}}$ for expedited order is 0 (an extension that $l_{i}^{\mathrm{e}}=l_{i}$ and $l_{i}^{\mathrm{r}}=l_{i}+1$ for a general non-negative $l_{i}$ is discussed in Section 5). The assumption that the leadtime difference between a regular and an expedited order must be 1 appears restrictive, but relaxing it makes the problem too complicated to yield an optimal control policy that is analytically solvable. This is because the resulting state space for each stage has to be augmented to include the pipeline inventory scheduled to arrive in future periods. In this case, it is known that, even for a single-stage system $N=1$, the optimal policy is complicated and state-dependent (Whittemore and Saunders [20]).

For each stage $i$, the unit expedited and regular order cost from stage $i+1$ is $k_{i}^{\mathrm{E}}$ and $k_{i}^{\mathrm{R}}$, respectively, with $k_{i}^{\mathrm{E}}>k_{i}^{\mathrm{R}}$. Echelon holding cost $h_{i}$ is incurred for each unit of on-hand inventory held in echelon $i$ per period, whereas backlog cost $p$ is incurred for each unit of backlog at stage 1 per period. The installation holding cost for stage $i$ is $H_{i}=\sum_{j=i}^{N} h_{j}$.

The following additional notation is needed.
$D(j)=j$-period demand $(j$-fold convolution of singleperiod demand as demands are i.i.d. across periods), $j=$ $1,2, \ldots$;

$F_{j}(\cdot)=$ the cumulative distribution function of $D(j)$, $j=1,2, \ldots$;

$\bar{F}_{j}(\cdot)=1-F_{j}(\cdot), j=1,2, \ldots ;$

$\alpha=$ the discount factor, i.e., $0 \leq \alpha<1$.

For notational simplicity, we use $D$ to denote a generic one-period demand and suppress the subscript of $F_{j}$ and $\bar{F}_{j}$ when $j=1$, i.e., $F(\cdot)=F_{1}(\cdot)$ and $\bar{F}(\cdot)=\bar{F}_{1}(\cdot)$.

The sequence of events is as follows. First, at the beginning of every period, each stage receives the regular order placed in the previous period. Second, starting from stage $N$, each stage places expedited and regular orders sequentially. Specifically, stage $N$ first places its expedited order from the outside supplier and receives it immediately. It then places a regular order which will be delivered at the beginning of next period (note that stage $N$ 's expedited order is immediately available to satisfy the order from stage $N-1$ ). Stage $N-1$ then decides its expedited and regular orders from stage $N$. Again, the expedited order stage $N-1$ received can be used to satisfy the order from stage $N-2$. This top-down ordering process continues until stage 1 places its expedited and regular orders from stage 2 . As an expedited order has a shipping leadtime 0 , the model allows expediting from any upstream stage to any downstream stage subject to inventory availability at the upstream stages. Finally, demand is realized during the period at stage 1 and all costs are incurred at the end of the period. The objective is to minimize the total discounted cost over an infinite planning horizon.

Lawson and Porteus [13] showed that the optimal policy of this problem ${ }^{1}$ is of a base-stock type, and that the optimal base-stock levels can be computed through a nested recursive algorithm.

First, let $c_{i}^{\mathrm{E}}=k_{i}^{\mathrm{E}}-k_{i}^{\mathrm{R}}+h_{i}$ and $c_{i}^{\mathrm{R}}=\alpha k_{i}^{\mathrm{E}}-k_{i}^{\mathrm{R}}$. Because $k_{i}^{\mathrm{E}}>k_{i}^{\mathrm{R}}$ and $h_{i} \geq 0, c_{i}^{\mathrm{E}}>0$. In addition, we assume $c_{i}^{\mathrm{R}} \geq 0$. If this is not the case, then the regular shipping mode will never be used and the model reduces to the Clark-Scarf model with a single supply mode between stages. To see that, suppose $c_{i}^{\mathrm{R}}<0$ or $\alpha k_{i}^{\mathrm{E}}<k_{i}^{\mathrm{R}}$, then it is more economical for stage $i$ to order the unit using expedited shipping in the next period rather than to order it at the current period using a regular order. (Lawson and Porteus [13] defined $c_{i}^{\mathrm{R}}=k_{i}^{\mathrm{R}}-\alpha k_{i}^{\mathrm{E}} \leq 0$, but here we keep the cost coefficient non-negative.) We call $c_{i}^{\mathrm{E}}$ the relative unit expedited ordering cost and $c_{i}^{\mathrm{R}}$ the relative unit regular ordering cost (in the rest of the article, "relative" is occasionally skipped for the sake of simplicity).

\footnotetext{
${ }^{1}$ Lawson and Porteus [13] also considered a so-called unit detained cost at stage $i$, which is assumed to be 0 in this article for the ease of exposition. Our results can easily be extended to include such a cost, if desired.
} 
We now present the computational algorithm for the optimal base-stock levels. Let $x^{-}=\max \{-x, 0\}$. Define $G_{1}^{\mathrm{E}}(y)=c_{1}^{\mathrm{E}} y+\left(H_{1}+p\right) \mathrm{E}\left[(y-D)^{-}\right]$, which is convex with minimizer $s_{1}^{\mathrm{E}}$. Let, for $i=1, \ldots, N$,

$$
\begin{aligned}
G_{i}^{\mathrm{R}}(y) & =-c_{i}^{\mathrm{R}} y+G_{i}^{\mathrm{E}}\left(y \wedge s_{i}^{\mathrm{E}}\right)+\alpha \mathrm{E}\left[G_{i}^{\mathrm{E}}\left((y-D) \vee s_{i}^{\mathrm{E}}\right)\right], \\
s_{i}^{\mathrm{R}} & =\arg \min _{y} G_{i}^{\mathrm{R}}(y),
\end{aligned}
$$

and, for $i=1, \ldots, N-1$,

$$
\begin{aligned}
G_{i+1}^{\mathrm{E}}(y) & =c_{i+1}^{\mathrm{E}} y+G_{i}^{\mathrm{R}}\left(y \wedge s_{i}^{\mathrm{R}}\right), \\
s_{i+1}^{\mathrm{E}} & =\arg \min _{y} G_{i+1}^{\mathrm{E}}(y),
\end{aligned}
$$

where both $G_{i}^{\mathrm{E}}(\cdot)$ and $G_{i}^{\mathrm{R}}(\cdot)$ are univariate convex functions. Here, we refer to $G_{i}^{\mathrm{E}}\left(y \wedge s_{i}^{\mathrm{E}}\right)$ and $G_{i}^{\mathrm{R}}\left(y \wedge s_{i}^{\mathrm{R}}\right)$ as the induced penalty function within stage $i$ and induced penalty function between stages $i$ and $i+1$, respectively. ${ }^{2}$ Note that the algorithm above does not directly lead to the minimum total discounted cost for the system. The optimal top-down echelon base-stock policy works as follows (see Ref. [13]). Starting from stage $N$, each stage tries to raise its echelon inventory level and position to the expedited base-stock level $s_{i}^{\mathrm{E}}$ and regular base-stock level $s_{i}^{\mathrm{R}}$, respectively, taking upstream decisions as given and ignoring downstream decisions.

Comparing (1)-(4) with the optimization algorithm of the Clark-Scarf system (see some details in Section 5), we note the following important differences. First, for each stage $i$, consider the regular base-stock level $s_{i}^{\mathrm{R}}$ as the base-stock level in the Clark-Scarf model. To compute $s_{i}^{\mathrm{R}}$, we need to first optimize an additional expedited ordering base-stock level $s_{i}^{\mathrm{E}}$ using (4). Second, due to the existence of two base-stock levels for each stage $i$, the ending inventory level of a period could be higher than its expedited base-stock level in the next period. This results in the third term in (1), which is not in the optimization algorithm of the Clark-Scarf model. It shows that the regular order decision of the current period depends on the expedited order decision of the next period, which then in turn influences its downstream stage's regular order. Thus, in the strict sense, the optimal policy is not "myopic" but one-period ahead and the expedited order decision cannot be simply regarded as an additional "stage" between two echelons of the Clark-Scarf model.

In the rest of this section, we present some structural properties on the optimal policies that will be used to derive simple bounds for the optimal base-stock levels.

\footnotetext{
${ }^{2}$ A more precise definition of the induced penalty functions should be $G_{i}^{\mathrm{E}}\left(y \wedge s_{i}^{\mathrm{E}}\right)-G_{i}^{\mathrm{E}}\left(s_{i}^{\mathrm{E}}\right)$ and $G_{i}^{\mathrm{R}}\left(y \wedge s_{i}^{\mathrm{R}}\right)-G_{i}^{\mathrm{R}}\left(s_{i}^{\mathrm{R}}\right)$; but since $G_{i}^{\mathrm{E}}\left(s_{i}^{\mathrm{E}}\right)$ and $G_{i}^{\mathrm{R}}\left(s_{i}^{\mathrm{R}}\right)$ are constant and will not affect the analysis, they are omitted.
}

PROPOSITION 1: (i) $s_{i}^{\mathrm{E}}<s_{i-1}^{\mathrm{R}}$, for $i=2, \ldots, N$. (ii) $s_{i}^{\mathrm{E}}<s_{i}^{\mathrm{R}}$, for $i=1, \ldots, N$.

PROOF: For part (i), note that $s_{i}^{\mathrm{E}}$ is determined by

$$
\left(G_{i}^{\mathrm{E}}(y)\right)^{\prime}=c_{i}^{\mathrm{E}}+\left(G_{i-1}^{\mathrm{R}}\left(y \wedge s_{i-1}^{\mathrm{R}}\right)\right)^{\prime}=0 .
$$

When $y=s_{i-1}^{\mathrm{R}}, c_{i}^{\mathrm{E}}+\left(G_{i-1}^{\mathrm{R}}\left(s_{i-1}^{\mathrm{R}}\right)\right)^{\prime}=c_{i}^{\mathrm{E}}>0$. Thus, it follows from the convexity of $G_{i}^{\mathrm{E}}(y)$ that $s_{i}^{\mathrm{E}}<s_{i-1}^{\mathrm{R}}$.

Similarly, for part (ii), note that $s_{i}^{\mathrm{R}}$ is the solution of

$$
\begin{aligned}
\left(G_{i}^{\mathrm{R}}(y)\right)^{\prime}=-c_{i}^{\mathrm{R}}+ & \left(G_{i}^{\mathrm{E}}\left(y \wedge s_{i}^{\mathrm{E}}\right)\right)^{\prime} \\
& +\alpha \mathrm{E}\left[G_{i}^{\mathrm{E}}\left((y-D) \vee s_{i}^{\mathrm{E}}\right)\right]^{\prime}=0 .
\end{aligned}
$$

Since, for $y \leq s_{i}^{\mathrm{E}},\left(G_{i}^{\mathrm{E}}\left(y \wedge s_{i}^{\mathrm{E}}\right)\right)^{\prime} \leq 0$ and $\mathrm{E}\left[G_{i}^{\mathrm{E}}((y-D) \vee\right.$ $\left.\left.s_{i}^{\mathrm{E}}\right)\right]^{\prime}=0$, we have $\left(G_{i}^{\mathrm{R}}(y)\right)^{\prime}<0$ as $c_{i}^{\mathrm{R}}>0$. The convexity of $G_{i}^{\mathrm{R}}(y)$ implies $s_{i}^{\mathrm{R}}>s_{i}^{\mathrm{E}}$.

In the following paragraphs, we develop solutions for the optimal base-stock levels $s_{i}^{\mathrm{E}}$ and $s_{i}^{\mathrm{R}}$, which solely depend on the demand distribution, which we term distribution-function solutions. By Eqs. (1)-(4), the optimal base-stock levels $s_{i}^{\mathrm{E}}$ and $s_{i}^{\mathrm{R}}$ are the solution of $\left(G_{i}^{\mathrm{E}}(y)\right)^{\prime}=0$ and $\left(G_{i}^{\mathrm{R}}(y)\right)^{\prime}=0$, respectively. For stage 1 , taking derivative of $G_{1}^{\mathrm{E}}(y)$ with respect to $y$ yields

$$
\left(G_{1}^{\mathrm{E}}(y)\right)^{\prime}=c_{1}^{\mathrm{E}}-\left(H_{1}+p\right) P(D>y)=0,
$$

hence the optimal expedited base-stock level for stage 1 is

$$
s_{1}^{\mathrm{E}}=\bar{F}^{-1}\left(\frac{c_{1}^{\mathrm{E}}}{H_{1}+p}\right) .
$$

Note that if $c_{1}^{\mathrm{E}}>H_{1}+p$, then $\left(G_{1}^{\mathrm{E}}(y)\right)^{\prime}>0$ from (7), which implies that $s_{1}^{\mathrm{E}}=-\infty$ and the expedited shipping is never used at stage 1 . So, in general for $j=1,2, \ldots$, we define $\bar{F}_{j}^{-1}(x)=-\infty$ for $x>1\left(F_{j}^{-1}(x)=-\infty\right.$ for $\left.x<0\right)$ and similarly $\bar{F}_{j}^{-1}(x)=\infty$ for $x<0\left(F_{j}^{-1}(x)=\infty\right.$ for $\left.x>1\right)$.

To solve $s_{1}^{\mathrm{R}}$, it follows from part (ii) of Proposition 1 that we only need to consider the solution of $\left(G_{1}^{\mathrm{R}}(y)\right)^{\prime}=0$ on $y \geq s_{1}^{\mathrm{E}}$. Thus, $s_{1}^{\mathrm{R}}$ is the solution of

$$
\begin{aligned}
& \left(G_{1}^{\mathrm{R}}(y)\right)^{\prime}=-c_{1}^{\mathrm{R}}+\alpha c_{1}^{\mathrm{E}} P\left(D \leq y-s_{1}^{\mathrm{E}}\right) \\
& \quad-\alpha\left(H_{1}+p\right) P\left(D \leq y-s_{1}^{\mathrm{E}}, D(2)>y\right)=0,
\end{aligned}
$$

in which $D(2)=D+D^{\prime}$ and $D^{\prime}$ is another single-period demand independent of $D$. For a single-stage system with two delivery modes, Eqs. (8) and (9) provide the optimal solutions, which can be easily computed. 
Applying (9) and Proposition 1, we obtain that $s_{2}^{\mathrm{E}}$ is the solution of

$$
\begin{aligned}
\left(G_{2}^{\mathrm{E}}(y)\right)^{\prime}=c_{2}^{\mathrm{E}}-c_{1}^{\mathrm{R}}+\left(c_{1}^{\mathrm{E}}-\left(H_{1}+p\right) P(D>y)\right) \mathbf{1}\left(y<s_{1}^{\mathrm{E}}\right) \\
+\alpha c_{1}^{\mathrm{E}} P\left(D \leq y-s_{1}^{\mathrm{E}}\right)-\alpha\left(H_{1}+p\right) \\
\quad \times P\left(D \leq y-s_{1}^{\mathrm{E}}, D(2)>y\right)=0, \quad(10)
\end{aligned}
$$

and that $s_{2}^{\mathrm{R}}$ is the solution of

$$
\begin{aligned}
& \left(G_{2}^{\mathrm{R}}(y)\right)^{\prime}= \\
& -c_{2}^{\mathrm{R}}+\alpha c_{2}^{\mathrm{E}} P\left(D \leq y-s_{2}^{\mathrm{E}}\right)-\alpha c_{1}^{\mathrm{R}}\left(D \leq y-s_{2}^{\mathrm{E}}, D>y-s_{1}^{\mathrm{R}}\right) \\
& \quad+\alpha c_{1}^{\mathrm{E}} P\left(D \leq y-s_{2}^{\mathrm{E}}, D>y-s_{1}^{\mathrm{E}}\right) \\
& +\alpha^{2} c_{1}^{\mathrm{E}} P\left(D \leq y-s_{2}^{\mathrm{E}}, D>y-s_{1}^{\mathrm{R}}, D(2) \leq y-s_{1}^{\mathrm{E}}\right) \\
& -\alpha\left(H_{1}+p\right) P\left(D \leq y-s_{2}^{\mathrm{E}}, D>y-s_{1}^{\mathrm{E}}, D(2)>y\right) \\
& -\alpha^{2}\left(H_{1}+p\right) P\left(D \leq y-s_{2}^{\mathrm{E}}, D>y-s_{1}^{\mathrm{R}}, D(2)\right. \\
& \left.\quad \leq y-s_{1}^{\mathrm{E}}, D(3)>y\right)=0 .
\end{aligned}
$$

It is clear that the distribution-function solutions for a twostage system with expedited ordering are more complicated than for a four-stage Clark-Scarf system (see Ref. [2]). This is mainly due to the last term in Eq. (1) of the computational algorithm, which further couples each stage's regular order and expedited order decisions between two consecutive periods. This process of deriving distribution-function solution can be continued for $s_{i}^{\mathrm{E}}$ and $s_{i}^{\mathrm{R}}$ for all $i$. The expression naturally becomes more and more complicated as $i$ increases.

PROPOSITION 2: For $i=2, \ldots, N, s_{i}^{\mathrm{E}} \geq s_{i-1}^{\mathrm{E}}$ if and only if $c_{i-1}^{\mathrm{R}} \geq c_{i}^{\mathrm{E}}$.

PROOF: Recall that $s_{i}^{\mathrm{E}}$ is the solution of

$$
\begin{aligned}
& \left(c_{i}^{\mathrm{E}}-c_{i-1}^{\mathrm{R}}\right)+\left(G_{i-1}^{\mathrm{E}}(y)\right)^{\prime} \mathbf{1}\left(y<s_{i-1}^{\mathrm{E}}\right) \\
& \quad+\alpha \mathrm{E}\left[\left(G_{i-1}^{\mathrm{E}}(y-D)\right)^{\prime} \mathbf{1}\left(y-D \geq s_{i-1}^{\mathrm{E}}\right)\right]=0 .
\end{aligned}
$$

We first show that if $c_{i-1}^{\mathrm{R}} \geq c_{i}^{\mathrm{E}}$, then $s_{i}^{\mathrm{E}} \geq s_{i-1}^{\mathrm{E}}$. Suppose $c_{i-1}^{\mathrm{R}} \geq c_{i}^{\mathrm{E}}$, then for any $y \leq s_{i-1}^{\mathrm{E}}$, the first two terms on the left hand side of (11) are nonpositive due to the convexity of $G_{i-1}^{\mathrm{E}}(y)$ and the optimality of $s_{i-1}^{\mathrm{E}}$; the third term is equal to 0 . Thus, because the left hand side of (11) is increasing in $y$, we conclude that $s_{i}^{\mathrm{E}} \geq s_{i-1}^{\mathrm{E}}$.

We next prove that, if $c_{i-1}^{\mathrm{R}}<c_{i}^{\mathrm{E}}$, then $s_{i}^{\mathrm{E}}<s_{i-1}^{\mathrm{E}}$. Substituting $y$ by $s_{i-1}^{\mathrm{E}}$ in (11), we obtain

$$
\begin{aligned}
& \left(c_{i}^{\mathrm{E}}-c_{i-1}^{\mathrm{R}}\right)+\left(G_{i-1}^{\mathrm{E}}(y)\right)^{\prime} \mathbf{1}\left(s_{i-1}^{\mathrm{E}}<s_{i-1}^{\mathrm{E}}\right) \\
& +\alpha \mathrm{E}\left[\left(G_{i-1}^{\mathrm{E}}\left(s_{i-1}^{\mathrm{E}}-D\right)\right)^{\prime} \mathbf{1}\left(s_{i-1}^{\mathrm{E}}-D \geq s_{i-1}^{\mathrm{E}}\right)\right]=c_{i}^{\mathrm{E}}-c_{i-1}^{\mathrm{R}}>0 .
\end{aligned}
$$

So again, because the left hand side of (11) is increasing in $y$, we must have $s_{i}^{\mathrm{E}}<s_{i-1}^{\mathrm{E}}$.
The intuition behind this result is as follows. Recall that $c_{i-1}^{\mathrm{R}}=\alpha k_{i-1}^{\mathrm{E}}-k_{i-1}^{\mathrm{R}}$, which can be regarded as the cost saving of stage $i-1$ by using a regular order of one unit from stage $i$ in a period instead of expediting one unit next period; $c_{i}^{\mathrm{E}}=k_{i}^{\mathrm{E}}-k_{i}^{\mathrm{R}}+h_{i}$ is the relative cost of stage $i$ between expediting one unit from stage $i+1$ that can be used immediately for stage $i-1$ and using regular shipping that arrives next period. From the perspective of the whole system, if the former cost saving is higher than the latter extra cost, then stage $i$ should keep some units available to meet regular ordering from stage $i-1$, i.e., $s_{i}^{\mathrm{E}}-s_{i-1}^{\mathrm{E}} \geq 0$; otherwise, it should not.

This result further provides the condition under which the optimal expedited base-stock levels are monotone with the stage index: If $c_{i-1}^{\mathrm{R}}>c_{i}^{\mathrm{E}}$ for all $i$, then $s_{N}^{\mathrm{E}} \geq s_{N-1}^{\mathrm{E}} \geq \cdots \geq$ $s_{1}^{\mathrm{E}}$. This result can simplify the distribution-function solution. For example, if $c_{1}^{\mathrm{R}} \geq c_{2}^{\mathrm{E}}$, then(10) is simplified to

$$
\begin{aligned}
& c_{2}^{\mathrm{E}}-c_{1}^{\mathrm{R}}+\alpha c_{1}^{\mathrm{E}} P\left(D \leq y-s_{1}^{\mathrm{E}}\right) \\
&-\alpha\left(H_{1}+p\right) P\left(D \leq y-s_{1}^{\mathrm{E}}, D(2)>y\right)=0 .
\end{aligned}
$$

PROPOSITION 3: For $i=2, \ldots, N$, if

$$
s_{i-1}^{\mathrm{R}}-s_{i}^{\mathrm{E}} \leq F^{-1}\left(\frac{c_{i}^{\mathrm{R}}}{\alpha c_{i}^{\mathrm{E}}}\right),
$$

then $s_{i}^{\mathrm{R}} \geq s_{i-1}^{\mathrm{R}}$.

PROOF: Since $s_{i}^{\mathrm{R}}$ is the solution of

$$
\begin{array}{r}
-c_{i}^{\mathrm{R}}+\alpha \mathrm{E}\left[\left(c_{i}^{\mathrm{E}}+\left(G_{i-1}^{\mathrm{R}}(y-D)\right)^{\prime} \mathbf{1}\left(y-D<s_{i-1}^{\mathrm{R}}\right)\right)\right. \\
\left.\left.\times \mathbf{1}\left(y-D \geq s_{i}^{\mathrm{E}}\right)\right)\right]=0,
\end{array}
$$

to show $s_{i}^{\mathrm{R}} \geq s_{i-1}^{\mathrm{R}}$, it is sufficient to prove

$$
-c_{i}^{\mathrm{R}}+\alpha \mathrm{E}\left[\left(c_{i}^{\mathrm{E}}+\left(G_{i-1}^{\mathrm{R}}\left(s_{i-1}^{\mathrm{R}}-D\right)\right)^{\prime}\right) \mathbf{1}\left(s_{i-1}^{\mathrm{R}}-D \geq s_{i}^{\mathrm{E}}\right)\right] \leq 0 .
$$

Note that for any possible sample path $D=d \geq 0$, $\left(G_{i-1}^{\mathrm{R}}\left(s_{i-1}^{\mathrm{R}}-d\right)\right)^{\prime} \leq 0$. So if $-c_{i}^{\mathrm{R}}+\alpha c_{i}^{\mathrm{E}} P\left(s_{i-1}^{\mathrm{R}}-D \geq s_{i}^{\mathrm{E}}\right) \leq$ 0 , or

$$
P\left(D \leq s_{i-1}^{\mathrm{R}}-s_{i}^{\mathrm{E}}\right) \leq \frac{c_{i}^{\mathrm{R}}}{\alpha c_{i}^{\mathrm{E}}},
$$

then the result is valid. The ratio $0 \leq c_{i}^{\mathrm{R}} / \alpha c_{i}^{\mathrm{E}} \leq 1$ is always satisfied by the definitions of $c_{i}^{\mathrm{R}}$ and $c_{i}^{\mathrm{E}}$.

The previous results not only provide some structural properties of the optimal base-stock levels but, more importantly, will also be used in the derivation of bounds for the optimal base-stock levels and computational heuristics in the following sections. 
We end this section with the following proposition that presents the comparative statics results of the optimal basestock levels.

PROPOSITION 4: (i) $s_{i}^{\mathrm{E}}$ is decreasing in $c_{j}^{\mathrm{E}}$ for $j \leq i$, independent of $c_{j}^{\mathrm{E}}$ for $j>i$, increasing in $c_{j}^{\mathrm{R}}$ for $j<i$, independent of $c_{j}^{\mathrm{R}}$ for $j \geq i$ and increasing in $p$.

(ii) $s_{i}^{\mathrm{R}}$ is decreasing in $c_{j}^{\mathrm{E}}$ for $j \leq i$, independent of $c_{j}^{\mathrm{E}}$ for $j>i$, increasing in $c_{j}^{\mathrm{R}}$ for $j \leq i$, independent of $c_{j}^{\mathrm{R}}$ for $j>i$ and increasing in $p$.

Proposition 4 shows that the impacts of system cost parameters on $s_{i}^{\mathrm{E}}$ and $s_{i}^{\mathrm{R}}$ are similar. This proposition is intuitive, as these two base-stock levels are complementary to each other. The result can be explained by the following lines of reasoning. For each stage $i$, if its relative expediting cost gets higher, then stage $i$ is less willing to use expedition, resulting in a lower expedited base-stock level $s_{i}^{\mathrm{E}}$. Similarly, if the relative expediting cost of stage $j, j<i$, becomes higher, then less expedited shipping would be used at stage $j$, with the result that the echelon expedited base-stock level of stage $i$ becomes lower. However, if the relative regular ordering cost of stage $i$ rises (the actual unit regular shipping cost is lower or the actual expedited shipping cost is higher), then stage $i$ would use more regular shipping, and hence, the regular echelon base-stock level would become higher. Thus, if stage $j$ 's, $j<i$, relative regular ordering cost becomes higher, then stage $j$ would tend to keep a higher regular base-stock level, leading indirectly to an increase in both the echelon expedited and regular base-stock levels at stage $i$. That $s_{i}^{\mathrm{R}}$ is decreasing in $c_{j}^{\mathrm{E}}, j<i$ is because, when the expediting cost of stage $j$ gets higher, fewer expedited orders would be placed and, as a result, stage $i$ would try not to keep as much inventory, leading to a lower echelon base-stock level for regular shipping. Finally, that both $s_{i}^{\mathrm{E}}$ and $s_{i}^{\mathrm{R}}$ increase with $p$ has its intuitive appeal: With a higher shortage cost, each stage should keep a higher (echelon) inventory level to avoid shortage.

\section{LOWER AND UPPER BOUNDS}

In this section, we develop several sets of newsvendor-type lower and upper bounds for the optimal echelon base-stock levels.

Before presenting the results, we first outline the basic ideas used in developing upper and lower bounds. Note that $s_{i}^{\mathrm{E}}$ is determined by $\left(G_{i}^{\mathrm{E}}(y)\right)^{\prime}=0$ and $\left(G_{i}^{\mathrm{E}}(y)\right)^{\prime}$ is an increasing function of $y$. If we can find a simple upper bound function $\bar{g}(y)$ such that $\left(G_{i}^{\mathrm{E}}(y)\right)^{\prime} \leq \bar{g}(y)$, then the solution of $\bar{g}(y)=0$ is a lower bound for $s_{i}^{\mathrm{E}}$. Similarly, if we can find another simple lower bound function $g(y)$ such that $\left(G_{i}^{\mathrm{E}}(y)\right)^{\prime} \geq \underline{g}(y)$, then the solution of $\underline{g}(y)=0$ is an upper bound for $s_{i}^{\mathrm{E}}$. The bounds for $s_{i}^{\mathrm{R}}$ can be analogously constructed. Moreover, the simpler and tighter the upper bound function $\bar{g}(y)$ (lower bound $\underline{g}(y))$ is to $\left(G_{i}^{\mathrm{E}}(y)\right)^{\prime}$, the simpler and better the resulting lower (upper) bound. Hence, the challenge is to find simple and tight bounding functions $\bar{g}(y)$ and $g(y)$. This idea was also adopted by Chao and Zhou [2] to derive bounds of the optimal base-stock levels for the ClarkScarf model. However, the addition of expedition option in each stage makes the construction and derivation of $g(y)$ and $\bar{g}(y)$ more complex and challenging here.

Since $s_{1}^{\mathrm{E}}$ is known in a closed form, we shall only develop bounds for $s_{i}^{\mathrm{E}}, i \geq 2$, and for $s_{i}^{\mathrm{R}}, i \geq 1$. Let $c_{0}^{\mathrm{R}}=0$. Note that, for $i=1,2, \ldots, N, \sum_{j=1}^{i}\left(c_{j}^{\mathrm{E}}-c_{j-1}^{\mathrm{R}}\right) \geq 0$ and $\sum_{j=1}^{i} \alpha^{i-j}\left(c_{j}^{\mathrm{E}}-c_{j-1}^{\mathrm{R}}\right) \geq 0$, since $c_{j}^{\mathrm{E}}-c_{j}^{\mathrm{R}}=(1-\alpha) k_{j}^{\mathrm{E}}+h_{j}>$ 0 and $\alpha c_{j}^{\mathrm{E}}-c_{j}^{\mathrm{R}}=(1-\alpha) k_{j}^{\mathrm{R}}+\alpha h_{j} \geq 0$.

Before we present the bounds, we give the following result that specifies conditions under which stage $i$ would never use the expedited shipping mode.

PROPOSITION 5: For $i=1, \ldots, N$,

(i) if $c_{i}^{\mathrm{E}}+\sum_{j=1}^{i-1}\left(\alpha c_{j}^{\mathrm{E}}-c_{j}^{\mathrm{R}}\right)>H_{1}+p$, then $s_{i}^{\mathrm{E}}=-\infty$; (ii) if $\sum_{j=1}^{i}\left(\alpha c_{j}^{\mathrm{E}}-c_{j}^{\mathrm{R}}\right)>H_{1}+p$, then $s_{j}^{\mathrm{R}}=-\infty$ and $s_{j}^{\mathrm{E}}=-\infty$ for $j \geq i$;

Therefore, in the following derivation of bounds, we assume $c_{i}^{\mathrm{E}}+\sum_{j=1}^{i-1}\left(\alpha c_{j}^{\mathrm{E}}-c_{j}^{\mathrm{R}}\right) \leq H_{1}+p$ and $\sum_{j=1}^{i}\left(\alpha c_{j}^{\mathrm{E}}-\right.$ $\left.c_{j}^{\mathrm{R}}\right) \leq H_{1}+p$ for all $i$. We first present three sets of newsvendor-type lower bounds.

THEOREM 1: For $i=1, \ldots, N$, the lower bounds for $s_{i}^{\mathrm{E}}$ and $s_{i}^{\mathrm{R}}$ are, respectively,

$$
\begin{array}{r}
\underline{s}_{i}^{\mathrm{E} 1}=\max \left\{\bar{F}^{-1}\left(\frac{\sum_{j=1}^{i}\left(c_{j}^{\mathrm{E}}-c_{j-1}^{\mathrm{R}}\right)}{H_{1}+p}\right),\right. \\
\left.\bar{F}^{-1}\left(\frac{\sum_{j=1}^{i} \alpha^{i-j}\left(c_{j}^{\mathrm{E}}-c_{j-1}^{\mathrm{R}}\right)}{\alpha^{i-1}\left(H_{1}+p\right)}\right)\right\},
\end{array}
$$

and

$$
\begin{array}{r}
\underline{s}_{i}^{\mathrm{R} 1}=\max \left\{\bar{F}^{-1}\left(\frac{-c_{i}^{\mathrm{R}}+\sum_{j=1}^{i}\left(c_{j}^{\mathrm{E}}-c_{j-1}^{\mathrm{R}}\right)}{H_{1}+p}\right),\right. \\
\left.\bar{F}^{-1}\left(\frac{-c_{i}^{\mathrm{R}}+\sum_{j=1}^{i} \alpha^{i-j+1}\left(c_{j}^{\mathrm{E}}-c_{j-1}^{\mathrm{R}}\right)}{\alpha^{i}\left(H_{1}+p\right)}\right)\right\} .
\end{array}
$$

As previously noted, the ratio within each pair of large parentheses is clearly non-negative. If the ratio is greater than 1 , the resulting lower bound is trivial, i.e., it is $-\infty$. 
We illustrate the proof of Theorem 1 using the first term in the brackets of $\underline{s}_{1}^{\mathrm{R} 1}$ and $\underline{s}_{2}^{\mathrm{E} 1}$. Applying the idea explained at the outset of this section, we need to find upper bound functions $\bar{g}(y)$ for $\left(G_{1}^{\mathrm{R}}(y)\right)^{\prime}$ and $\left(G_{2}^{\mathrm{E}}(y)\right)^{\prime}$. Note that

$$
\begin{aligned}
& \left(G_{1}^{\mathrm{R}}(y)\right)^{\prime} \\
& =-c_{1}^{\mathrm{R}}+\left(G_{1}^{\mathrm{E}}(y)\right)^{\prime} \mathbf{1}\left(y<s_{1}^{\mathrm{E}}\right)+\alpha \mathrm{E}\left[\left(G_{1}^{\mathrm{E}}\right)^{\prime}\left((y-D) \vee s_{1}^{\mathrm{E}}\right)\right] \\
& \leq-c_{1}^{\mathrm{R}}+\left(G_{1}^{\mathrm{E}}(y)\right)^{\prime} \mathbf{1}\left(y<s_{1}^{\mathrm{E}}\right)+\left(G_{1}^{\mathrm{E}}\right)^{\prime}\left(y \vee s_{1}^{\mathrm{E}}\right) \\
& =-c_{1}^{\mathrm{R}}+\left(G_{1}^{\mathrm{E}}(y)\right)^{\prime} \\
& =-c_{1}^{\mathrm{R}}+c_{1}^{\mathrm{E}}-\left(H_{1}+p\right) P(D>y) \\
& =\bar{g}(y),
\end{aligned}
$$

where the inequality follows from $0 \leq \alpha<1$ and the convexity of $G_{1}^{\mathrm{E}}(y)$. Hence, the solution of $\bar{g}(y)=0$ or $\bar{F}^{-1}\left(\left(-c_{1}^{\mathrm{R}}+c_{1}^{\mathrm{E}}\right) /\left(H_{1}+p\right)\right)$ is a lower bound for $s_{1}^{\mathrm{R}}$.

Now consider $s_{2}^{\mathrm{E}}$. We have

$$
\begin{aligned}
\left(G_{2}^{\mathrm{E}}(y)\right)^{\prime} & =c_{2}^{\mathrm{E}}+\left(G_{1}^{\mathrm{R}}\left(y \wedge s_{1}^{\mathrm{R}}\right)\right)^{\prime} \\
& \leq c_{2}^{\mathrm{E}}+\left(G_{1}^{\mathrm{R}}\left(y \wedge s_{1}^{\mathrm{R}}\right)\right)^{\prime}+\left(G_{1}^{\mathrm{R}}\left(y \vee s_{1}^{\mathrm{R}}\right)\right)^{\prime} \\
& \leq c_{2}^{\mathrm{E}}-c_{1}^{\mathrm{R}}+c_{1}^{\mathrm{E}}-\left(H_{1}+p\right) P(D>y) \\
& =\sum_{j=1}^{2}\left(c_{j}^{\mathrm{E}}-c_{j-1}^{\mathrm{R}}\right)-\left(H_{1}+p\right) P(D>y) \\
& =\bar{g}(y),
\end{aligned}
$$

where the first inequality follows from $\left(G_{1}^{\mathrm{R}}\left(y \vee s_{1}^{\mathrm{R}}\right)\right)^{\prime} \geq 0$ and the second inequality follows from (14). Thus, the solution of $\bar{g}(y)=0$ or $\bar{F}^{-1}\left(\sum_{j=1}^{2}\left(c_{j}^{\mathrm{E}}-c_{j-1}^{\mathrm{R}}\right) /\left(H_{1}+p\right)\right)$ is a lower bound for $s_{2}^{\mathrm{E}}$. The complete proof can be done by mathematical induction, which we provide in the Appendix.

Before we provide some intuition behind the derivation of this set of lower bounds, we first define $\left(G_{i}^{\mathrm{E}}\left(y \wedge s_{i}^{\mathrm{E}}\right)\right)^{\prime}$ and $\left(G_{i}^{\mathrm{R}}\left(y \wedge s_{i}^{\mathrm{R}}\right)\right)^{\prime}$ as the marginal induced penalty cost within and between stages respectively; and $\mathrm{E}\left[\left(G_{i}^{\mathrm{E}}\right)^{\prime}\left((y-D) \vee s_{i}^{\mathrm{E}}\right)\right]$ as the expected marginal cost from the next period due to the order of the current period. To derive $\underline{s}_{i}^{R 1}$, we ignore one period demand to amplify the marginal cost increment of the regular order of this period to the next, so that stage $i$ tends to keep a lower regular base-stock level. For $\underline{s}_{i}^{E 1}$, we essentially impose an additional marginal cost $\left(G_{i-1}^{\mathrm{R}}\left(y \vee s_{i-1}^{\mathrm{R}}\right)\right)^{\prime}$ on the expedited order, and stage $i$ therefore would set a lower expedited base-stock level.

On the basis of Theorem 1, we can develop another set of lower bounds. We first define, for $i=1, \ldots, N$,

$$
\begin{aligned}
& A_{i, j}=-c_{i}^{\mathrm{E}}+B_{i-1, j}, \quad j=1, \ldots, i-1, \\
& B_{i, j}=c_{i}^{\mathrm{R}}-\alpha A_{i, j}^{-}, j=1, \ldots, i,
\end{aligned}
$$

in which $A_{i, i}=0$ and $A_{i, j}^{-}=\max \left\{-A_{i, j}, 0\right\}$.
The computation of $A_{i, j}$ and $B_{i, j}$ is as follows. First, $A_{1,1}=0$ and $B_{1,1}$ is computed from (16). Inductively, suppose $A_{i, j}$ and $B_{i, j}$ have been computed for a given $i$ and all $j \leq i$. Then, using $B_{i, j}$ and (15) we can compute $A_{i+1, j}$ for $j=1, \ldots, i$, and $A_{i+1, i+1}=0$. And using (16), we can compute $B_{i+1, j}$ for $j=1, \ldots, i+1$.

THEOREM 2: If $\sum_{j=1}^{i} \alpha^{i-j}\left(c_{j}^{\mathrm{E}}-c_{j-1}^{\mathrm{R}}\right) \leq \alpha^{i-1}\left(H_{1}+\right.$ $p$ ), for $i=2, \ldots, N$, then,

$$
\underline{s}_{i}^{\mathrm{E} 2}=\max \left\{F_{k}^{-1}\left(\frac{A_{i, i-k+1}}{\sum_{j=1}^{i-k+1} \alpha^{i-j}\left(c_{j}^{\mathrm{E}}-c_{j-1}^{\mathrm{R}}\right)}\right), k=2, \ldots, i\right\}
$$

is a lower bound for $s_{i}^{\mathrm{E}}$, and for $i=1, \ldots, N$,

$$
\begin{array}{r}
\underline{s}_{i}^{\mathrm{R} 2}=\max \left\{F_{k+1}^{-1}\left(\frac{B_{i, i-k+1}}{\sum_{j=1}^{i-k+1} \alpha^{i-j+1}\left(c_{j}^{\mathrm{E}}-c_{j-1}^{\mathrm{R}}\right)}\right),\right. \\
k=1, \ldots, i\}
\end{array}
$$

is a lower bound for $s_{i}^{\mathrm{R}}$.

Note that the terms in the brackets of (17) and (18) actually present a sequence of lower bounds for each stage $i$. To obtain this sequence of lower bounds for $s_{i}^{\mathrm{R}}$, we drop $G_{j}^{\mathrm{E}}\left(y \wedge s_{j}^{\mathrm{E}}\right)$ sequentially from $j=i$ to $j=1$ in (6). In other words, we use a smaller induced penalty cost from downstream stage which results in a lower base-stock level. For $\underline{s}_{i}^{\mathrm{E} 2}$, we use the same idea as that for deriving $\underline{s}_{i}^{\mathrm{E} 1}$. As for the ratios in each pair of parentheses, we can show they are always less than 1 . If the ratio is negative, the resulting lower bound is $-\infty$.

For each stage $i$, by ignoring the induced penalty cost $G_{i}^{\mathrm{E}}\left(y \wedge s_{i}^{\mathrm{E}}\right)$ within that stage and increasing the marginal cost increment from the next period due to the regular order of the current period, stage $i$, therefore, would set lower basestock levels. This idea is applied to derive the following set of lower bounds. Recall that $c_{i}^{\mathrm{R}} \leq \alpha c_{i}^{\mathrm{E}}$ for all $i$, so the ratios in the next theorem are always between 0 and 1 .

THEOREM 3: For $i=2, \ldots, N$, if $c_{i-1}^{\mathrm{R}}-c_{i}^{\mathrm{E}} \geq 0$, then

$$
\underline{s}_{i}^{\mathrm{E} 3}=s_{i-1}^{\mathrm{E}}+F^{-1}\left(\frac{c_{i-1}^{\mathrm{R}}-c_{i}^{\mathrm{E}}}{\alpha c_{i-1}^{\mathrm{E}}}\right)
$$

is a lower bound for $s_{i}^{\mathrm{E}}$. And for $i=1, \ldots, N$,

$$
\underline{s}_{i}^{\mathrm{R} 3}=s_{i}^{\mathrm{E}}+F^{-1}\left(\frac{c_{i}^{\mathrm{R}}}{\alpha c_{i}^{\mathrm{E}}}\right)
$$

is a lower bound for $s_{i}^{\mathrm{R}}$. 
Proposition 3 shows that if $c_{i}^{\mathrm{E}} \leq c_{i-1}^{\mathrm{R}}$, a lower bound for $s_{i}^{\mathrm{E}}$ is $s_{i-1}^{\mathrm{E}}$. Theorem 3 provides a sharper lower bound for $s_{i}^{\mathrm{E}}$ under this condition. Although this bound depends on the optimal $s_{i-1}^{\mathrm{E}}$ (and $s_{i}^{\mathrm{E}}$ for the lower bound of $s_{i}^{\mathrm{R}}$ ), we can use the largest available lower bounds for $s_{i-1}^{\mathrm{E}}$ and $s_{i}^{\mathrm{E}}$ to obtain newsvendor-type bounds for $\underline{s}_{i}^{E 3}$ and $\underline{s}_{i}^{R 3}$. Furthermore, note from Proposition 3 that, unless $c_{i}^{\mathrm{E}^{i}} \leq c_{i-1}^{\mathrm{R}}$, the lower bounds for $s_{i}^{\mathrm{E}}$ cannot be written as the lower bound of the optimal expedited base-stock level of its downstream plus a non-negative number. However, from Proposition 1, the lower bound of $s_{i}^{\mathrm{R}}$ can always be written as the sum of $s_{i}^{\mathrm{E}}$ and a non-negative number.

We have presented three sets of lower bounds for the optimal expedited and regular base-stock levels. These lower bounds do not have a dominating relationship. That is, any lower bound can be a better one, depending on the problem instance. We will provide some discussion on the performance of different bounds in the numerical studies section.

We next present three sets of newsvendor-type upper bounds by constructing different lower bound functions for $\left(G_{i}^{\mathrm{E}}(y)\right)^{\prime}$ and $\left(G_{i}^{\mathrm{R}}(y)\right)^{\prime}$. The following set of upper bounds is developed by using a smaller marginal induced penalty cost within stage $\left(G_{i}^{\mathrm{E}}\left(y \wedge s_{i}^{\mathrm{E}}\right)\right)^{\prime}$.

THEOREM 4: For $i=1, \ldots, N$, the upper bounds for $s_{i}^{\mathrm{E}}$ and $s_{i}^{\mathrm{R}}$ are, respectively:

$$
\bar{s}_{i}^{\mathrm{E} 1}=\bar{F}_{i}^{-1}\left(\frac{c_{i}^{\mathrm{E}}-c_{i-1}^{\mathrm{R}}+\alpha c_{i-1}^{\mathrm{E}}}{H_{1}+p-\sum_{j=1}^{i-2}\left(\alpha c_{j}^{\mathrm{E}}-c_{j}^{\mathrm{R}}\right)}\right) ;
$$

and

$$
\bar{s}_{i}^{\mathrm{R} 1}=\bar{F}_{i+1}^{-1}\left(\frac{\alpha c_{i}^{\mathrm{E}}-c_{i}^{\mathrm{R}}}{\alpha\left(H_{1}+p-\sum_{j=1}^{i-1}\left(\alpha c_{j}^{\mathrm{E}}-c_{j}^{\mathrm{R}}\right)\right)}\right) .
$$

Note that our assumption on cost parameters following Proposition 5 guarantees that the ratios in the newsvendor bounds above are between 0 and 1 .

Again, we use $\bar{s}_{1}^{\mathrm{R} 1}$ and $\bar{s}_{2}^{\mathrm{E} 1}$ to demonstrate the derivation of the upper bounds. To derive $\bar{s}_{1}^{\mathrm{R} 1}$, we first note the following inequalities.

$$
\begin{aligned}
\left(G_{1}^{\mathrm{R}}(y)\right)^{\prime}= & -c_{1}^{\mathrm{R}}+\left(G_{1}^{\mathrm{E}}(y)\right)^{\prime} \mathbf{1}\left(y \leq s_{1}^{\mathrm{E}}\right) \\
& +\alpha \mathrm{E}\left[\left(G_{1}^{\mathrm{E}}(y-D)\right)^{\prime} \mathbf{1}\left(y-D \geq s_{1}^{\mathrm{E}}\right)\right] \\
\geq & -c_{1}^{\mathrm{R}}+\mathrm{E}\left[\left(G_{1}^{\mathrm{E}}(y-D)\right)^{\prime} \mathbf{1}\left(y-D<s_{1}^{\mathrm{E}}\right)\right] \\
& +\alpha \mathrm{E}\left[\left(G_{1}^{\mathrm{E}}(y-D)\right)^{\prime} \mathbf{1}\left(y-D \geq s_{1}^{\mathrm{E}}\right)\right]
\end{aligned}
$$

$$
\begin{aligned}
& =-c_{1}^{\mathrm{R}}+c_{1}^{\mathrm{E}} P\left(y-D<s_{1}^{\mathrm{E}}\right) \\
& \quad-\left(H_{1}+p\right) P\left(D(2)>y, y-D<s_{1}^{\mathrm{E}}\right) \\
& +\alpha c_{1}^{\mathrm{E}} P\left(y-D \geq s_{1}^{\mathrm{E}}\right) \\
& \quad-\alpha\left(H_{1}+p\right) P\left(D(2)>y, y-D \geq s_{1}^{\mathrm{E}}\right) \\
& \quad \geq-c_{1}^{\mathrm{R}}+\alpha c_{1}^{\mathrm{E}}-\left(H_{1}+p\right) P(D(2)>y),
\end{aligned}
$$

where the first inequality follows from that, by the convexity of $G_{1}^{\mathrm{E}}(y)$,

$$
\left(G_{1}^{\mathrm{E}}(y)\right)^{\prime} \mathbf{1}\left(y \leq s_{1}^{\mathrm{E}}\right) \geq \mathrm{E}\left[\left(G_{1}^{\mathrm{E}}(y-D)\right)^{\prime} \mathbf{1}\left(y-D<s_{1}^{\mathrm{E}}\right)\right] .
$$

Clearly, setting Eqn. (23) to 0 gives an upper bound of $s_{1}^{\mathrm{R}}$. But we can obtain a better bound by observing that $\left(G_{1}^{\mathrm{E}}(y)\right)^{\prime} \mathbf{1}\left(y \leq s_{1}^{\mathrm{E}}\right)=0$ for $y \geq s_{1}^{\mathrm{E}}$. Hence, as $s_{1}^{\mathrm{R}} \geq s_{1}^{\mathrm{E}}$ from Proposition 1, we have

$$
\begin{array}{r}
-c_{1}^{\mathrm{R}}+\alpha \mathrm{E}\left[\left(G_{1}^{\mathrm{E}}(y-D)\right)^{\prime} \mathbf{1}\left(y-D \geq s_{1}^{\mathrm{E}}\right)\right] \geq-c_{1}^{\mathrm{R}}+\alpha c_{1}^{\mathrm{E}} \\
-\alpha\left(H_{1}+p\right) P(D(2)>y)=\underline{g}(y),
\end{array}
$$

and solving $\underline{g}(y)=0$ we obtain $\bar{s}_{1}^{\mathrm{R} 1}$. To derive $\bar{s}_{2}^{\mathrm{E} 1}$, we apply the inequality (23),

$$
\begin{aligned}
& \left(G_{2}^{\mathrm{E}}(y)\right)^{\prime}=c_{2}^{\mathrm{E}}+\left(G_{1}^{\mathrm{R}}(y)\right)^{\prime} \mathbf{1}\left(y<s_{i}^{\mathrm{R}}\right) \\
& \geq c_{2}^{\mathrm{E}}+\left[-c_{1}^{\mathrm{R}}+\alpha c_{1}^{\mathrm{E}}-\left(H_{1}+p\right) P(D(2)>y)\right] \mathbf{1}\left(y<s_{1}^{\mathrm{R}}\right) \\
& \quad \geq c_{2}^{\mathrm{E}}-\left(H_{1}+p-\left(\alpha c_{1}^{\mathrm{E}}-c_{1}^{\mathrm{R}}\right)\right) P(D(2)>y)=\underline{g}(y),
\end{aligned}
$$

where the second inequality follows from $-c_{1}^{\mathrm{R}}+\alpha c_{1}^{\mathrm{E}} \geq 0$. Thus, the solution of $\underline{g}(y)=0$ is an upper bound of $s_{2}^{\mathrm{E}}$, which is $\bar{s}_{2}^{\mathrm{E} 1}$.

The second set of upper bounds is obtained by replacing $\mathrm{E}\left[\left(G_{i}^{\mathrm{E}}(y-D)\right)^{\prime} 1\left(y-D \geq s_{i}^{\mathrm{E}}\right)\right]$ in (6) for stage $i$ by $\mathrm{E}\left[\left(G_{i}^{\mathrm{E}}(y-D)\right)^{\prime}\right]$, i.e., reducing the expected marginal cost from the next period due to the current period's regular order.

THEOREM 5: For $i=2, \ldots, N$,

$$
\bar{s}_{i}^{\mathrm{E} 2}=\bar{s}_{i-1}^{\mathrm{R} 2},
$$

is an upper bound for $s_{i}^{\mathrm{E}}$, and, let $s_{0}^{\mathrm{R}}=0$, for $i=1, \ldots, N$,

$$
\begin{aligned}
\bar{s}_{i}^{\mathrm{R} 2} & =s_{i-1}^{\mathrm{R}} \\
& +\bar{F}^{-1}\left(\frac{\alpha c_{i}^{\mathrm{E}}-c_{i}^{\mathrm{R}}}{\min \left\{\alpha c_{i}^{\mathrm{E}}, \alpha\left(H_{1}+p-\sum_{j=1}^{i-1}\left(\alpha c_{j}^{\mathrm{E}}-c_{j}^{\mathrm{R}}\right)\right)\right\}}\right)
\end{aligned}
$$

is an upper bound for $s_{i}^{\mathrm{R}}$. 
Again, to compute (25), the available smallest upper bound of $s_{i-1}^{\mathrm{R}}$ is used instead of the optimal one. In particular, repetitive applications of Theorem 5 yield the following bounds. is

COROLLARY 1: One set of upper bounds for $s_{i}^{\mathrm{E}}$ and $s_{i}^{\mathrm{R}}$

$$
\begin{aligned}
\bar{s}_{i}^{\mathrm{E}} & =\sum_{\ell=1}^{i-1} \\
& \bar{F}^{-1}\left(\frac{\alpha c_{\ell}^{\mathrm{E}}-c_{\ell}^{\mathrm{R}}}{\min \left\{\alpha c_{\ell}^{\mathrm{E}}, \alpha\left(H_{1}+p-\sum_{j=1}^{\ell-1}\left(\alpha c_{j}^{\mathrm{E}}-c_{j}^{\mathrm{R}}\right)\right)\right\}}\right), \\
\bar{s}_{i}^{\mathrm{R}} & =\sum_{\ell=1}^{i} \bar{F}^{-1}\left(\frac{\alpha c_{\ell}^{\mathrm{E}}-c_{\ell}^{\mathrm{R}}}{\min \left\{\alpha c_{\ell}^{\mathrm{E}}, \alpha\left(H_{1}+p-\sum_{j=1}^{\ell-1}\left(\alpha c_{j}^{\mathrm{E}}-c_{j}^{\mathrm{R}}\right)\right)\right\}}\right) .
\end{aligned}
$$

We now develop another set of upper bounds for the optimal base-stock levels by replacing the marginal induced penalty cost between stages $\left(G_{i}^{\mathrm{R}}\left(y \wedge s_{i}^{\mathrm{R}}\right)\right)^{\prime}$ with a smaller one (see details in the Appendix).

Let $C_{i}=c_{i}^{\mathrm{E}}-c_{i-1}^{\mathrm{R}}-C_{i-1}^{-}, i=1, \ldots, N$, with $C_{0}=0$.

THEOREM 6: For $i=1, \ldots, N$,

$$
\begin{aligned}
\bar{s}_{i}^{\mathrm{E} 3}=\min & \left\{\bar{F}^{-1}\left(\frac{C_{i}}{H_{1}+p}\right),\right. \\
& \left.\bar{F}_{2}^{-1}\left(\frac{c_{i}^{\mathrm{E}}-c_{i-1}^{\mathrm{R}}+\min \left\{\alpha C_{i-1}, C_{i-1}\right\}}{H_{1}+p}\right)\right\}
\end{aligned}
$$

is an upper bound for $s_{i}^{\mathrm{E}}$, and

$$
\bar{s}_{i}^{\mathrm{R} 3}=\bar{F}_{2}^{-1}\left(\frac{-c_{i}^{\mathrm{R}}+\alpha C_{i}}{\alpha\left(H_{1}+p\right)}\right)
$$

is an upper bound for $s_{i}^{\mathrm{R}}$.

As in the case of lower bounds, none of the upper bounds developed above dominates the others. That is, any one of these upper bounds can be sharper, depending on the problem instance.

\section{GENERAL LEADTIMES}

In the previous sections, the leadtimes for regular and expedited ordering are assumed to be 1 and 0 , respectively. In this section, we extend the results to the case where leadtimes for regular and expedited ordering are $l_{i}^{\mathrm{r}}=l_{i}+1$ and $l_{i}^{\mathrm{e}}=l_{i}$, respectively, with $l_{i}$ being an arbitrary non-negative integer. Clearly, this represents an extension of Fukuda's model [10] to serial multi-echelon systems. This extension can be obtained from the model in Section 3 by inserting stages to represent units of leadtime. Specifically, we can represent each of the $l_{i}$ units of leadtime as an auxiliary stage with only regular shipping mode, zero ordering cost, and zero echelon holding cost. Under such a cost structure, once an expedited or a regular shipping is initiated at a nonauxiliary stage, there is no incentive to keep it in the auxiliary stages. Mathematically, this is equivalent to setting the optimal echelon base-stock levels for these auxiliary stages at infinity.

The bottom-up recursive algorithm for computing the optimal echelon base-stock levels for the more general leadtime case is as follows. Redefine, for $i=1, \ldots, N$,

$$
\begin{aligned}
c_{i}^{\mathrm{E}} & =k_{i}^{\mathrm{E}}-k_{i}^{\mathrm{R}}+\alpha^{l_{i}} h_{i}, \\
c_{i}^{\mathrm{R}} & =\alpha k_{i}^{\mathrm{E}}-k_{i}^{\mathrm{R}} .
\end{aligned}
$$

Let

$$
G_{1}^{\mathrm{E}}(y)=c_{1}^{\mathrm{E}} y+\alpha^{l_{1}}\left(H_{1}+p\right) \mathrm{E}\left[\left(y-D\left(l_{1}+1\right)\right)^{-}\right],
$$

and let $s_{1}^{\mathrm{E}}$ be the minimizer of $G_{1}^{\mathrm{E}}(y)$. For $i=1, \ldots, N$, compute

$$
G_{i}^{\mathrm{R}}(y)=-c_{i}^{\mathrm{R}} y+G_{i}^{\mathrm{E}}\left(y \wedge s_{i}^{\mathrm{E}}\right)+\alpha \mathrm{E}\left[G_{i}^{\mathrm{E}}\left((y-D) \vee s_{i}^{\mathrm{E}}\right)\right],
$$

$$
s_{i}^{\mathrm{R}}=\arg \min _{y} G_{i}^{\mathrm{R}}(y),
$$

and for $i=1, \ldots, N-1$,

$$
\begin{aligned}
G_{i+1}^{\mathrm{E}}(y) & =c_{i+1}^{\mathrm{E}} y+\alpha^{l_{i+1}} \mathrm{E}\left[G_{i}^{\mathrm{R}}\left(\left(y-D\left(l_{i+1}\right)\right) \wedge s_{i}^{\mathrm{R}}\right)\right], \\
s_{i+1}^{\mathrm{E}} & =\arg \min _{y} G_{i+1}^{\mathrm{E}}(y) .
\end{aligned}
$$

The algorithm above can be derived from the following argument. First, note that the results of the previous sections can be easily extended to the case where some stages only have one transportation mode available. Recall that for the Clark-Scarf model with only one transportation mode (suppose the unit ordering cost is $k_{i}^{\mathrm{R}}, i=1, \ldots, N$ ) and leadtimes between stages being 1, the optimal echelon base-stock levels are computed as follows (for a detailed derivation see Ref. [2]).

$G_{1}(y)=(1-\alpha) k_{1}^{\mathrm{R}}+\alpha h_{1} y+\alpha\left(H_{1}+p\right) \mathrm{E}\left[(y-D(2))^{-}\right]$, 
and $s_{1}^{*}=\arg \min G_{1}(y)$; for $i>1$,

$$
\begin{aligned}
G_{i-1}^{i}(y) & =G_{i-1}\left(y \wedge s_{i-1}^{*}\right) \\
G_{i}(y) & =(1-\alpha) k_{i}^{\mathrm{R}}+\alpha h_{i} y+\alpha \mathrm{E}\left[G_{i-1}^{i}(y-D)\right], \\
s_{i}^{*} & =\arg \min G_{i}(y),
\end{aligned}
$$

in which $s_{i}^{*}$ is the optimal base-stock level. Therefore, if, for example, stage $i$ only has one transportation mode with leadtime 1 , whereas stages $i-1$ and $i+1$ have two transportation modes with leadtimes 0 and 1 , then the optimal echelon basestock level $s_{i}^{*}$ for stage $i$ is obtained by optimizing $G_{i}$, where $G_{i}$ is calculated as above by taking $G_{i-1}$ as $G_{i-1}^{\mathrm{R}}$ of the previous sections and $s_{i-1}^{*}$ taking value $s_{i-1}^{\mathrm{R}} ; G_{i+1}^{\mathrm{E}}(y)$ is calculated from Eq. (3), taking $G_{i}$ as $G_{i}^{\mathrm{R}}$ and $s_{i}^{\mathrm{R}}$ as $s_{i}^{*}$.

We now illustrate how to apply this idea to derive Eqs. (28)-(31) for a serial system with $l_{i}^{\mathrm{e}}=l_{i}$ and $l_{i}^{\mathrm{r}}=l_{i}+1$, $i=1, \ldots, N$. Insert $l_{1}$ stages before the original stage 1 , each of which only has the regular transportation mode with leadtime 1 . We call these $l_{1}$ stages added stage 1 , added stage $2, \ldots$, added stage $l_{1}$ from downstream to upstream. The added stage 1 faces customer demand and is allocated a shortage cost $p$ and echelon holding cost 0 . We denote the added stage $i$ by a subscript $(a, i)$. Therefore, $G_{(a, 1)}(y)=$ $\alpha\left(H_{1}+p\right) \mathrm{E}\left[(y-D(2))^{-}\right]$with a minimizer being infinity, and as a result we have $G_{(a, 1)}^{2}(y)=G_{(a, 1)}(y)$ and $G_{(a, 2)}(y)=$ $\alpha \mathrm{E}\left[G_{(a, 1)}^{2}(y-D)\right]=\alpha^{2}\left(H_{1}+p\right) \mathrm{E}\left[(y-D(3))^{-}\right]$. This process continues until the original stage 1 is reached and we obtain

$$
\begin{aligned}
G_{1}^{\mathrm{E}}(y)=c_{1}^{\mathrm{E}} y+ & G_{\left(a, l_{1}\right)}(y) \\
& =c_{1}^{\mathrm{E}} y+\alpha^{l_{1}}\left(H_{1}+p\right) \mathrm{E}\left[\left(y-D\left(l_{1}+1\right)\right)^{-}\right],
\end{aligned}
$$

where the first equality is due to zero leadtime for the expedited shipping at stage 1 and the minimizer of the added stage $l_{1}$ is infinity. It should be noted that we have $\alpha^{l_{1}} h_{1}$ in $c_{1}^{\mathrm{E}}$ since the holding cost at original stage 1 will incur $l_{1}$ periods later when the order arrives at the added auxiliary stage 1 . Applying algorithm (3) for the original stage 1, we obtain

$$
G_{1}^{\mathrm{R}}(y)=-c_{1}^{\mathrm{R}} y+G_{1}^{\mathrm{E}}\left(y \wedge s_{1}^{\mathrm{E}}\right)+\alpha \mathrm{E}\left[G_{1}^{\mathrm{E}}\left((y-D) \vee s_{1}^{\mathrm{E}}\right)\right] .
$$

This process continues and we obtain that, for stage $i+1$,

$$
G_{i+1}^{\mathrm{E}}(y)=c_{i+1}^{\mathrm{E}} y+\alpha^{l_{i+1}} \mathrm{E}\left[G_{i}^{\mathrm{R}}\left(\left(y-D\left(l_{i+1}\right)\right) \wedge s_{i}^{\mathrm{R}}\right)\right],
$$

in which $G_{i}^{\mathrm{R}}(y)$ is computed from the added $l_{i}$ stage before stage $i$, and $G_{i+1}^{\mathrm{R}}$ remains to be given by Eq. (3).

On the basis of the above algorithm, it is clear that Proposition 1 (ii) and Propositions 2-4 in Section 3 continue to hold under this more general leadtime setting. Proposition 1 (i) does not hold in general because when applying the analogous argument as the proof of Proposition $1, s_{i}^{\mathrm{E}}$ may be greater than $s_{i-1}^{\mathrm{R}}$ since the solution of $c_{i}^{\mathrm{E}}+\mathrm{E}\left[\left(G_{i-1}^{\mathrm{R}}\right)^{\prime}((y-\right.$ $\left.\left.\left.D\left(l_{i}\right)\right) \wedge s_{i-1}^{\mathrm{R}}\right)\right]=0$ may be greater than $s_{i-1}^{\mathrm{R}}$. (In the previous analysis, however, $D\left(l_{i}\right)=0$.)

We now demonstrate the changes for each set of lower and upper bounds under this more general setting of leadtimes. We omit the detailed derivation because it is analogous to the analysis of the previous sections with the algorithm (28)-(31). Let $L_{(i, j)}=\sum_{k=i}^{j} l_{k}$.

The first set of lower bounds with general leadtimes is, for $i=1,2, \ldots, N$

$$
\begin{aligned}
\underline{s}_{i}^{E 1}=\max & \left\{\bar{F}_{L_{(1, i)}+1}^{-1}\left(\frac{c_{i}^{\mathrm{E}}+\sum_{j=1}^{i-1} \alpha^{L_{(j+1, i)}}\left(c_{j}^{\mathrm{E}}-c_{j}^{\mathrm{R}}\right)}{\alpha^{L_{(1, i)}}\left(H_{1}+p\right)}\right),\right. \\
& \left.\bar{F}_{L_{(1, i)}+1}^{-1}\left(\frac{c_{i}^{\mathrm{E}}+\sum_{j=1}^{i-1} \alpha^{L_{(j+1, i)}+i-j}\left(c_{j}^{\mathrm{E}}-c_{j}^{\mathrm{R}}\right)}{\alpha^{L_{(1, i)}+i-1}\left(H_{1}+p\right)}\right)\right\},
\end{aligned}
$$

and

$$
\begin{aligned}
\underline{s}_{i}^{\mathrm{R} 1}=\max \{ & \bar{F}_{L_{(1, i)}+1}^{-1}\left(\frac{\sum_{j=1}^{i} \alpha^{L_{(j+1, i)}}\left(c_{j}^{\mathrm{E}}-c_{j}^{\mathrm{R}}\right)}{\alpha^{L_{(1, i)}}\left(H_{1}+p\right)}\right), \\
& \left.\bar{F}_{L_{(1, i)}+1}^{-1}\left(\frac{\sum_{j=1}^{i} \alpha^{L_{(j+1, i)}+i-j+1}\left(c_{j}^{\mathrm{E}}-c_{j}^{\mathrm{R}}\right)}{\alpha^{L_{(1, i)}+i}\left(H_{1}+p\right)}\right)\right\},
\end{aligned}
$$

where it is clear that the ratio within each pair of parentheses is non-negative from the definition of $c_{i}^{\mathrm{E}}$ and $c_{i}^{\mathrm{R}}$. Again, if the ratio is larger than 1 , from our previous definition, the lower bound is $-\infty$.

To generalize the second set of lower bounds presented in Theorems 2, we first need to redefine for $i=1, \ldots, N$,

$$
\begin{aligned}
& A_{i, j}=-c_{i}^{\mathrm{E}}+\alpha^{l_{i}} B_{i-1, j}, \quad j=1, \ldots, i-1, \\
& B_{i, j}=c_{i}^{\mathrm{R}}-\alpha A_{i, j}^{-}, \quad j=1, \ldots, i,
\end{aligned}
$$

in which $A_{i, i}=0$ and $A_{i, j}^{-}=\max \left\{-A_{i, j}, 0\right\}$. If $\sum_{j=1}^{i} \alpha^{L_{(j+1, i)}+i-j}\left(c_{j}^{\mathrm{E}}-c_{j-1}^{\mathrm{R}}\right) \leq \alpha^{L_{(1, i)}+i-1}\left(H_{1}+p\right)$, for $i=2, \ldots, N$, then,

$$
\begin{aligned}
& \underline{s}_{i}^{\mathrm{E} 2}=\max \left\{F_{L_{(1, i)}+k}^{-1}\left(\frac{A_{i, i-k+1}}{\sum_{j=1}^{i-k+1} \alpha^{L_{(j+1, i)}+i-j}\left(c_{j}^{\mathrm{E}}-c_{j-1}^{\mathrm{R}}\right)}\right),\right. \\
& k=2, \ldots, i\}
\end{aligned}
$$


is a lower bound for $s_{i}^{\mathrm{E}}$, and for $i=1, \ldots, N$,

$$
\begin{aligned}
& \underline{s}_{i}^{\mathrm{R} 2}=\max \left\{F_{L_{(1, i)}+k+1}^{-1}\left(\frac{B_{i, i-k+1}}{\sum_{j=1}^{i-k+1} \alpha^{L_{(j+1, i)}+i-j+1}\left(c_{j}^{\mathrm{E}}-c_{j-1}^{\mathrm{R}}\right)}\right),\right. \\
& k=1, \ldots, i\}
\end{aligned}
$$

The last set of lower bounds here is, for $i=1, \ldots, N$,

$$
\underline{s}_{i}^{\mathrm{E} 3}=s_{i-1}^{\mathrm{E}}+F_{l_{i}+1}^{-1}\left(\frac{\alpha^{l_{i}} c_{i-1}^{\mathrm{R}}-c_{i}^{\mathrm{E}}}{\alpha^{l_{i}+1} c_{i-1}^{\mathrm{E}}}\right) .
$$

The expression of $\underline{s}_{i}^{\mathrm{R} 3}$ is the same as (20) because the leadtime difference between the expedited and regular shipping is still 1 .

The upper bounds with general leadtimes need to be modified more carefully because we can no longer apply part (i) of Proposition 1 as we did in the previous derivation.

Consider the first set of upper bounds. As $s_{i}^{\mathrm{E}}$ may not be less than $s_{i-1}^{\mathrm{R}}$, the upper bound for $s_{i}^{\mathrm{E}}$ becomes, for $i=1,2, \ldots, N$

$\bar{s}_{i}^{\mathrm{E} 1}=\bar{F}_{L_{(1, i)}+i}^{-1}\left(\frac{c_{i}^{\mathrm{E}}}{\alpha^{L_{(1, i)}}\left(H_{1}+p\right)-\sum_{j=1}^{i-1} \alpha^{L_{(j, i)}}\left(\alpha c_{j}^{\mathrm{E}}-c_{j}^{\mathrm{R}}\right)}\right)$.

For the upper bound $\bar{s}_{i}^{\mathrm{R} 1}$, as $s_{i}^{\mathrm{E}} \leq s_{i}^{\mathrm{R}}$ is still true, we have

$\bar{s}_{i}^{\mathrm{R} 1}=$

$\bar{F}_{L_{(1, i)}+i+1}^{-1}\left(\frac{\alpha c_{i}^{\mathrm{E}}-c_{i}^{\mathrm{R}}}{\alpha\left(\alpha^{L_{(1, i)}}\left(H_{1}+p\right)-\sum_{j=1}^{i-1} \alpha^{L_{(j, i)}}\left(\alpha c_{j}^{\mathrm{E}}-c_{j}^{\mathrm{R}}\right)\right)}\right)$.

For the second set of the upper bounds, $\bar{s}_{i}^{\mathrm{E} 2}$ is no longer valid. But we still have $\bar{s}_{i}^{\mathrm{R} 2}$, which is modified as follows.

$$
\begin{aligned}
& \bar{s}_{i}^{\mathrm{R} 2}=s_{i-1}^{\mathrm{R}} \\
& +\bar{F}_{l_{i}+1}^{-1}\left(\frac{\alpha c_{i}^{\mathrm{E}}-c_{i}^{\mathrm{R}}}{\alpha\left(\alpha^{L_{(1, i)}}\left(H_{1}+p\right)-\sum_{j=1}^{i-1} \alpha^{L_{(j, i)}}\left(\alpha c_{j}^{\mathrm{E}}-c_{j}^{\mathrm{R}}\right)\right)}\right) .
\end{aligned}
$$

Finally, for the last set of upper bounds, redefine $C_{i}=$ $c_{i}^{\mathrm{E}}-\alpha^{l_{i}}\left(c_{i-1}^{\mathrm{R}}-C_{i-1}^{-}\right), i=1, \ldots, N$, with $C_{0}=0$ and $C_{i-1}^{-}=\max \left\{-C_{i-1}, 0\right\}$. For $i=1, \ldots, N$,

$$
\bar{s}_{i}^{\mathrm{E} 3}=\bar{F}_{L_{(1, i)}+1}^{-1}\left(\frac{C_{i}}{\alpha^{L_{(1, i)}}\left(H_{1}+p\right)}\right),
$$

and

$$
\bar{s}_{i}^{\mathrm{R} 3}=\bar{F}_{L_{(1, i)}+2}^{-1}\left(\frac{-c_{i}^{\mathrm{R}}+\alpha C_{i}}{\alpha^{L_{(1, i)}+1}\left(H_{1}+p\right)}\right) .
$$

Note that we no longer have the second term in the brackets of (26) for the last set of upper bounds.

\section{HEURISTICS AND NUMERICAL STUDIES}

In this section, we develop a simple heuristic for the optimal echelon base-stock levels based on the lower and upper bounds obtained in the preceding sections. We also conduct a numerical study to demonstrate the effectiveness of the heuristic. We first focus on the case with $l_{i}^{\mathrm{e}}=0$. For computational purposes, the demand distribution is assumed to be discrete in this section.

For $i=1,2, \ldots, N$, let

$$
\begin{aligned}
& {\underset{s}{s}}_{i}^{\mathrm{E}}=\max \left\{\underline{s}_{i}^{\mathrm{E} j}, j=1,2,3\right\},{\underset{\sim}{s}}_{i}^{\mathrm{R}}=\max \left\{\underline{s}_{i}^{\mathrm{R} j}, j=1,2,3\right\} ; \\
& \tilde{s}_{i}^{\mathrm{E}}=\min \left\{\bar{s}_{i}^{\mathrm{E} j}, j=1,2,3\right\}, \tilde{s}_{i}^{\mathrm{R}}=\min \left\{\bar{s}_{i}^{\mathrm{R} j}, j=1,2,3\right\} .
\end{aligned}
$$

It is clear that ${\underset{\sim}{s}}_{i}^{\mathrm{E}} \leq s_{i}^{\mathrm{E}} \leq \tilde{s}_{i}^{\mathrm{E}}$ and ${\underset{\sim}{s}}_{i}^{\mathrm{R}} \leq s_{i}^{\mathrm{R}} \leq \tilde{s}_{i}^{\mathrm{R}}$, for $i=1, \ldots, N$. Furthermore, it follows from Proposition 2 that if $c_{i-1}^{\mathrm{R}} \leq c_{i}^{\mathrm{E}}$, then $s_{i}^{\mathrm{E}} \leq s_{i-1}^{\mathrm{E}}$. Hence, in the following, if $\tilde{s}_{i}^{\mathrm{E}}>\tilde{s}_{i-1}^{\mathrm{E}}$, then we set $\tilde{s}_{i}^{\mathrm{E}}=\tilde{s}_{i-1}^{\mathrm{E}}$; and if ${\underset{i}{s}}_{i}^{\mathrm{E}}>{\underset{i-1}{s}}_{i}^{\mathrm{E}}$, we set ${\underset{i}{s}}_{i-1}^{\mathrm{E}}={\underset{\sim}{s}}_{i}^{\mathrm{E}}$.

For $i \stackrel{i}{=} 1,2 \ldots, N$ and $0 \leq \beta \leq 1$, set

$$
\begin{aligned}
s_{i}^{\mathrm{E} h} & =\left[\beta{\underset{\sim}{s}}_{i}^{\mathrm{E}}+(1-\beta) \tilde{s}_{i}^{\mathrm{E}}\right], \\
s_{i}^{\mathrm{R} h} & =\left[\beta{\underset{\sim}{s}}_{i}^{\mathrm{R}}+(1-\beta) \tilde{s}_{i}^{\mathrm{R}}\right],
\end{aligned}
$$

in which [ ] is the round off operator. We choose $\beta=0.5$ as the heuristic policy. The heuristic policy works in exactly the same manner as the original top-down echelon base-stock policy, but $s_{i}^{\mathrm{E} h}$ and $s_{i}^{\mathrm{R} h}$ are used as the echelon base-stock levels for stage $i$.

In the following, we conduct a numerical study to test the effectiveness of the heuristic. We consider a three-stage $N=3$ system. The system parameters for the examples are $p \in\{30,60\}, h_{i} \in\{0.1,1\}, k_{i}^{\mathrm{E}} \in\{4,10\}, k_{i}^{\mathrm{R}} \in\{2,6\}$, for $i=1,2,3$, and $\alpha=0.95$. We present two groups of numerical examples classified by the demand distributions.

We use the relative error on the optimal system cost as the measure for the effectiveness of the heuristic. Let $\mathbf{x}=\left(x_{1}, \ldots, x_{N}\right)$ with $x_{i}$ being the initial echelon inventory level at stage $i$. Denote $v(\mathbf{x})$ and $\hat{v}(\mathbf{x})$ as the optimal cost and the cost of the heuristic policy with a given $\mathbf{x}$, respectively. To calculate these costs, we use successive approximation with a planing horizon $T=100$, and we observe that the total discounted cost converges in our numerical results. The relative error of the heuristic is defined as

$$
\text { Error } \%=\max _{-200 \leq x_{i} \leq 200, i=1, \ldots, N}\left\{\frac{\hat{v}(\mathbf{x})-v(\mathbf{x})}{v(\mathbf{x})} \times 100 \%\right\},
$$

in which, to avoid the influence of the initial state on the performance of the heuristic, we consider a reasonable large 

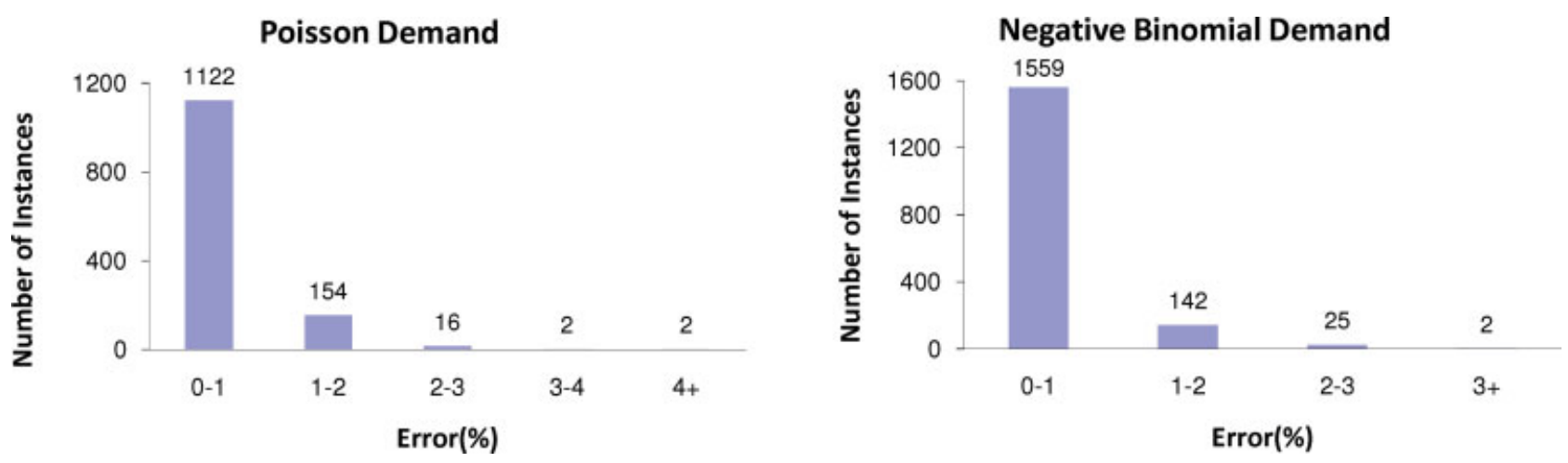

Figure 1. Performance summary of the heuristic: $l_{1}^{\mathrm{e}}=l_{2}^{\mathrm{e}}=l_{3}^{\mathrm{e}}=0$. [Color figure can be viewed in the online issue, which is available at www.interscience.wiley.com.]

number of possible combinations of initial echelon inventory levels, i.e., $x_{i} \in[-200,200]$ for all $i$. Figure 1 reports the distribution of errors with respect to the number of instances for these two groups of examples.

In Group 1, demand follows Poisson distribution with parameter $\lambda \in\{5,10,50\}$. By restricting $k_{i}^{\mathrm{E}}>k_{i}^{\mathrm{R}}$, we generate 432 instances by different combinations of the system parameters for each demand rate. The average relative error among 432 instances for $\lambda=5$ is $0.57 \%$ with the maximum $3.06 \%$, for $\lambda=10$ is $0.52 \%$ with the maximum $4.28 \%$, and for $\lambda=50$ is $0.33 \%$ with the maximum $1.70 \%$. The average relative error for all 1296 instances is $0.47 \%$. From these results, it can be seen that the average performance of the heuristic gets better when $\lambda$ increases.

The demand distribution for the second group of numerical examples is Negative Binomial with four sets of different mean and variance $(30,40),(30,120),(6,8)$, and $(6,24)$. This allows us to observe the impact of demand variance on the performance of the heuristic. The coefficient of variation for each set is $0.21,0.37,0.47$, and 0.82 correspondingly. Each pair of demand mean and variance with different system cost combinations also generates four sets of numerical

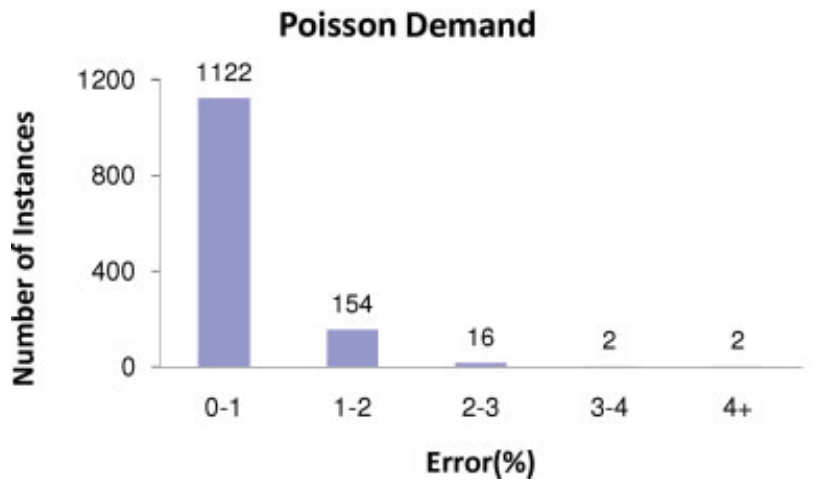

examples and each set includes 432 instances. The average relative error among 432 instances for the first set is $0.37 \%$ with the maximum $2.65 \%$, for the second is $0.42 \%$ with the maximum $3.62 \%$, for the third is $0.48 \%$ with the maximum $2.88 \%$, and for the fourth is $0.49 \%$ with the maximum $2.64 \%$. The average relative error for all 1728 instances is $0.44 \%$. We observe that the average performance of the heuristic is better with a smaller coefficient of variation. This result is intuitive, as a deviation from the optimal solution would cause a larger cost if the demand is more variable.

For the system in which $l_{i}^{\mathrm{e}}=l_{i}$ and $l_{i}^{\mathrm{r}}=l_{i}+1$ with $l_{i}>0$, a heuristic can be similarly derived once we have the bounds presented in Section 5. To see how the resulting heuristic ( $\beta=0.5$ ) performs, we test the preceding two groups of examples except that the leadtimes are now $l_{1}^{\mathrm{e}}=3, l_{2}^{\mathrm{e}}=1$, and $l_{3}^{\mathrm{e}}=3$ for the expedited orders and $l_{i}^{\mathrm{r}}=l_{i}^{\mathrm{e}}+1$. When demand is Poisson, for each of the three sets of instances differentiated by the demand rate, the average and maximum errors are $(0.56 \%, 2.11 \%),(0.42 \%, 1.57 \%)$, and $(0.20 \%$, $1.10 \%)$. When demand is Negative Binomial, the average and maximum errors for each of the four sets of problems are summarized as: $(0.38 \%, 1.27 \%)$; $(0.28 \%, 1.24 \%) ;(0.43 \%$,

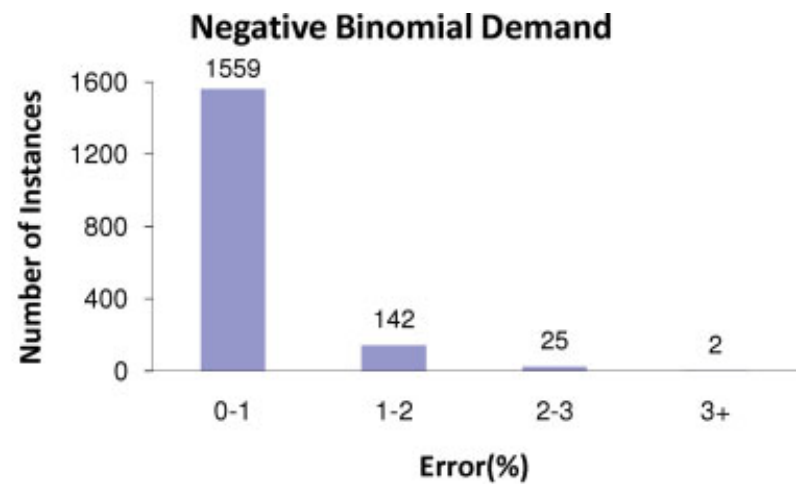

Figure 2. Performance summary of the heuristic: $l_{1}^{\mathrm{e}}=l_{3}^{\mathrm{e}}=3, l_{2}^{\mathrm{e}}=1$. [Color figure can be viewed in the online issue, which is available at www.interscience.wiley.com.]

Naval Research Logistics DOI 10.1002/nav 
$1.44 \%)$; and $(0.58 \%, 2.67 \%)$. These numerical results show that the heuristic also works well under this more general leadtime setting. We report the distribution of errors with respect to the number of instances in Fig. 2.

We conclude this section with some observations from our numerical results on the relative performance of different lower and upper bounds reported in Section 4. We find that, for lower bounds, in most cases, the first set of lower bound $\underline{s}_{i}^{\mathrm{E} 1}$ for expedited order and the third set of lower bound $\underline{s}_{i}^{\mathrm{R} 3}$ for regular order serve as the best lower bounds in our numerical examples. For instance, among 432 instances of the third set of Group 2 examples, 432 of ${\underset{\sim}{2}}_{2}^{\mathrm{E}}$ are from $\underline{s}_{2}^{\mathrm{E} 1}$ and 414 of ${\underset{\sim}{3}}_{3}^{\mathrm{E}}$ are from $\underline{s}_{3}^{\mathrm{E} 1} ; 324$ of $\underline{\sim}_{1}^{\mathrm{R}}$ are from $\underline{s}_{1}^{\mathrm{R} 3}, 339$ of ${\underset{\sim}{2}}_{2}^{\mathrm{R}}$ are from $\underline{s}_{2}^{\mathrm{R} 3}{ }^{3}$, and 350 of ${\underset{\sim}{3}}_{3}^{\mathrm{R}}$ are from $\underline{s}_{3}^{\mathrm{R} 3}$. With respect to upper bounds, the first set of upper bound $\bar{s}_{i}^{\mathrm{E} 1}$ and the third set of upper bound $\bar{s}_{i}^{E 3}$ for expedited base-stock levels show a similar performance, and are better than the second set of upper bound $\bar{s}_{i}^{\mathrm{E} 2}$; for the regular base-stock level, the first set of upper bound $\bar{s}_{i}^{\mathrm{R} 1}$ is the most effective one. But in general, no clear pattern seems to emerge on the impact of system parameters on bounds. We also test the effectiveness of bounds with different value of $\alpha$. We find that when $\alpha$ gets smaller, $\bar{s}_{i}^{\mathrm{E} 3}$ and $\bar{s}_{i}^{\mathrm{R} 3}$ get better (closer to the optimal ones), $\underline{s}_{i}^{\mathrm{E} 3}$ becomes worse.

\section{CONCLUSION}

In this article, we study an infinite-horizon, periodicreview, serial production/inventory system with expedited and regular shipping modes available between stages. We derive structural properties of the optimal policies and develop newsvendor-type lower and upper bounds for the optimal echelon base-stock levels. Among the different sets of lower and upper bounds, some perform better than others under different system parameters. These bounds lead to a simple and effective heuristic for the optimal inventory control policy. Numerical studies show that the heuristic performs well. We generalize the computational algorithm and other results to the case where expedited and regular orders in each stage $i$ have leadtimes $l_{i}$ and $l_{i}+1$, respectively, for an arbitrary non-negative integer $l_{i}$. The bounds and heuristic policies are given in closed forms in terms of cumulative demand distribution functions and primitive system parameters, hence, they immediately reveal the impact of the system parameters on the control policies. We believe that these results shed lights on the structure of the optimal policies for multi-echelon serial inventory systems with dual shipping modes, and on their implementability.

\section{APPENDIX}

In this appendix, we give the proofs for Propositions 4, 5, and Theorems $1-6$. In these proofs, the exchange of expectation and derivative is justified by Leibniz's rule.
PROOF OF PROPOSITION 4: In this proof, we denote the density function of a generic one-period demand by $f(\cdot)$. It follows from the definition and the convexity of $G_{i}^{\mathrm{E}}(y)$ and $G_{i}^{\mathrm{R}}(y)$ that $s_{i}^{\mathrm{E}}$ is decreasing in $c_{i}^{\mathrm{E}}$ and $s_{i}^{\mathrm{R}}$ is increasing in $c_{i}^{\mathrm{R}}$. To show that $s_{i}^{\mathrm{R}}$ is decreasing in $c_{i}^{\mathrm{E}}$, it suffices to prove that $\left(G_{i}^{\mathrm{R}}(y)\right)^{\prime}$ is increasing in $c_{i}^{\mathrm{E}}$. To that end, let $\left(G_{i}^{\mathrm{R}}(y)\right)^{\prime}=0$ be written as $g\left(y, c_{i}^{\mathrm{E}}\right)=0$, and let $s_{i}^{\mathrm{R}}\left(c_{i}^{\mathrm{E}}\right)$ be its solution. Recall that $g\left(y, c_{i}^{\mathrm{E}}\right)$ is increasing in $y$. Suppose $c_{i}^{\mathrm{E}} \leq c_{i}^{E^{\prime}}$. If $g\left(y, c_{i}^{\mathrm{E}}\right)$ is increasing in $c_{i}^{\mathrm{E}}$, then

$$
g\left(s_{i}^{\mathrm{R}}\left(c_{i}^{\mathrm{E}^{\prime}}\right), c_{i}^{\mathrm{E}}\right) \leq g\left(s_{i}^{\mathrm{R}}\left(c_{i}^{\mathrm{E}^{\prime}}\right), c_{i}^{\mathrm{E}^{\prime}}\right)=0 .
$$

Hence, it follows from $g\left(y, c_{i}^{\mathrm{E}}\right)$ is increasing in $y$ that, $s_{i}^{\mathrm{R}}\left(c_{i}^{\mathrm{E}}\right)$, determined by $g\left(y, c_{i}^{\mathrm{E}}\right)=0$, satisfies $s_{i}^{\mathrm{R}}\left(c_{i}^{\mathrm{E}}\right) \geq s_{i}^{\mathrm{R}}\left(c_{i}^{E^{\prime}}\right)$. This shows that $s_{i}^{\mathrm{R}}$ is decreasing in $c_{i}^{\mathrm{E}}$.

Note that

$g\left(y, c_{i}^{\mathrm{E}}\right)=-c_{i}^{\mathrm{R}}+\left(G_{i}^{\mathrm{E}}(y)\right)^{\prime} \mathbf{1}\left(y \leq s_{i}^{\mathrm{E}}\right)+\alpha \int_{0}^{y-s_{i}^{\mathrm{E}}}\left(G_{i}^{\mathrm{E}}(y-t)\right)^{\prime} d F(t)$.

Noting $\left(G_{i}^{\mathrm{E}}\right)_{y, c_{i}^{\mathrm{E}}}^{\prime \prime}(y)=1$, where $\left(G_{i}^{\mathrm{E}}\right)_{y, c_{i}^{\mathrm{E}}}^{\prime \prime}(y)$ represents the cross derivative with respective to $y$ and $c_{i}^{\mathrm{E}}$, we obtain

$$
\begin{aligned}
g_{c_{i}^{\mathrm{E}}}^{\prime}\left(y, c_{i}^{\mathrm{E}}\right)= & \mathbf{1}\left(y<s_{i}^{\mathrm{E}}\right)+\left(G_{i}^{\mathrm{E}}(y)\right)^{\prime}\left(\mathbf{1}\left(y<s_{i}^{\mathrm{E}}\right)\right)_{c_{i}^{\mathrm{E}}}^{\prime} \mathbf{1}\left(y<s_{i}^{\mathrm{E}}\right) \\
& +\alpha F\left(y-s_{i}^{\mathrm{E}}\right)-\alpha\left(G_{i}^{\mathrm{E}}\left(s_{i}^{\mathrm{E}}\right)\right)^{\prime} f\left(y-s_{i}^{\mathrm{E}}\right)\left(s_{i}^{\mathrm{E}}\right)_{c_{i}^{\mathrm{E}}}^{\prime} \\
= & \mathbf{1}\left(y<s_{i}^{\mathrm{E}}\right)+\left(G_{i}^{\mathrm{E}}(y)\right)^{\prime}\left(\mathbf{1}\left(y<s_{i}^{\mathrm{E}}\right)\right)_{c_{i}^{\mathrm{E}}}^{\prime} \mathbf{1}\left(y<s_{i}^{\mathrm{E}}\right) \\
& +\alpha F\left(y-s_{i}^{\mathrm{E}}\right) \\
\geq & 0
\end{aligned}
$$

where the second equality follows from $s_{i}^{\mathrm{E}}$ being the minimizer of $G_{i}^{\mathrm{E}}$, and the inequality follows from

$$
\left(G_{i}^{\mathrm{E}}(y)\right)^{\prime} \leq 0 \quad \text { when } y<s_{i}^{\mathrm{E}} \text {, and }\left(\mathbf{1}\left(y<s_{i}^{\mathrm{E}}\right)\right)_{c_{i}^{\mathrm{E}}}^{\prime} \leq 0
$$

because $s_{i}^{\mathrm{E}}$ is decreasing in $c_{i}^{\mathrm{E}}$. We next show that both $s_{i}^{\mathrm{E}}$ and $s_{i}^{\mathrm{R}}$ are decreasing in $c_{j}^{\mathrm{E}}$ for $j<i$. Suppose $\left(G_{i}^{\mathrm{E}}(y)\right)_{y, c_{j}^{\mathrm{E}}}^{\prime \prime} \geq 0$ for $i$, then for $i+1$, first take derivative of $G_{i}^{\mathrm{R}}$ with respect to $y$,

$$
\left(G_{i}^{\mathrm{R}}(y)\right)^{\prime}=-c_{i}^{\mathrm{R}}+\alpha \int_{0}^{y-s_{i}^{\mathrm{E}}}\left(G_{i}^{\mathrm{E}}(y-\xi)\right)^{\prime} d F(\xi)
$$

and then take derivative with respect to $c_{j}^{E}$,

$$
\left(G_{i}^{\mathrm{R}}(y)\right)_{y, c_{j}^{\mathrm{E}}}^{\prime \prime}=\alpha \int_{0}^{y-s_{i}^{\mathrm{E}}}\left(G_{i}^{\mathrm{E}}(y-\xi)\right)_{y, c_{j}^{\mathrm{E}}}^{\prime \prime} d F(\xi) \geq 0,
$$

which implies that $s_{i}^{\mathrm{R}}$ is decreasing in $c_{j}^{\mathrm{E}}$ for $j<i$. For $s_{i+1}^{\mathrm{E}}$, which is the solution of

$$
\left(G_{i+1}^{\mathrm{E}}(y)\right)^{\prime}=c_{i+1}^{\mathrm{E}}+\left(G_{i}^{\mathrm{R}}(y)\right)^{\prime}=0
$$

and from the previous analysis, it is clear that

$$
\left(G_{i+1}^{\mathrm{E}}(y)\right)_{y, c_{j}^{\mathrm{E}}}^{\prime \prime}=\left(G_{i}^{\mathrm{R}}(y)\right)_{y, c_{j}^{\mathrm{E}}}^{\prime \prime} \geq 0,
$$

which implies that $s_{i+1}^{\mathrm{E}}$ is decreasing in $c_{j}^{\mathrm{E}}$ for $j<i$. 
As $s_{1}^{\mathrm{E}}$ is independent of $c_{1}^{\mathrm{R}}$, we first prove $s_{2}^{\mathrm{E}}$ is increasing in $c_{1}^{\mathrm{R}}$. From Eq. (10), we have

$$
\left(G_{2}^{\mathrm{E}}(y)\right)_{y, c_{1}^{\mathrm{R}}}^{\prime \prime}=-1<0
$$

which implies that $s_{2}^{\mathrm{E}}$ is increasing in $c_{1}^{\mathrm{R}}$. Now suppose $\left(G_{i}^{\mathrm{E}}(y)\right)_{y, c_{j}^{\mathrm{R}}}^{\prime \prime}<0$ for $j<i$, then for $s_{i}^{\mathrm{R}}$,

$$
\left(G_{i}^{\mathrm{R}}(y)\right)_{y, c_{j}^{\mathrm{R}}}^{\prime \prime}=\alpha \int_{0}^{y-s_{i}^{\mathrm{E}}}\left(G_{i}^{\mathrm{E}}(y-\xi)\right)_{y, c_{j}^{\mathrm{R}}}^{\prime \prime} d F(\xi)<0
$$

and

$$
\left(G_{i+1}^{\mathrm{E}}(y)\right)_{y, c_{j}^{\mathrm{R}}}^{\prime \prime}=\left(G_{i}^{\mathrm{R}}(y)\right)_{y, c_{j}^{\mathrm{R}}}^{\prime \prime}<0 .
$$

Therefore, both $s_{i}^{\mathrm{E}}$ and $s_{i}^{\mathrm{R}}$ are increasing in $c_{j}^{\mathrm{R}}$ for $j<i$.

The proof of the result that both $s_{i}^{\mathrm{E}}$ and $s_{i}^{\mathrm{R}}$ are increasing in $p$ follows similar steps and we leave it for interested reader.

PROOF OF PROPOSITION 5: First notice that, if $\sum_{j=1}^{i}\left(\alpha c_{j}^{\mathrm{E}}-c_{j}^{\mathrm{R}}\right)>$ $H_{1}+p$, then $c_{k}^{\mathrm{E}}+\sum_{j=1}^{k-1}\left(\alpha c_{j}^{\mathrm{E}}-c_{j}^{\mathrm{R}}\right)>H_{1}+p$ for $k \geq i$.

To show part $(i)$, we need to first show that for all $i$,

$$
\left(G_{i}^{\mathrm{E}}(y)\right)^{\prime} \geq c_{i}^{\mathrm{E}}-\left(H_{1}+p-\sum_{j=1}^{i-1}\left(\alpha c_{j}^{\mathrm{E}}-c_{j}^{\mathrm{R}}\right)\right) P(D(i)>y) .
$$

If Eq. (32) is valid and if $c_{i}^{\mathrm{E}}+\sum_{j=1}^{i-1}\left(\alpha c_{j}^{\mathrm{E}}-c_{j}^{\mathrm{R}}\right)>H_{1}+p$ for some $i$, then $\left(G_{i}^{\mathrm{E}}(y)\right)^{\prime}>0$, and so, $s_{i}^{\mathrm{E}}=-\infty$.

Moreover, from Eq. (32)

$$
\begin{aligned}
\left(G_{i}^{\mathrm{R}}(y)\right)^{\prime}= & -c_{i}^{\mathrm{R}}+\left(G_{i}^{\mathrm{E}}(y)\right)^{\prime} \mathbf{1}\left(y \leq s_{i}^{\mathrm{E}}\right) \\
& +\alpha \mathrm{E}\left[\left(G_{i}^{\mathrm{E}}(y-D)\right)^{\prime} \mathbf{1}\left(y-D \geq s_{i}^{\mathrm{E}}\right)\right] \\
\geq & -c_{i}^{\mathrm{R}}+\mathrm{E}\left[\left(G_{i}^{\mathrm{E}}(y-D)\right)^{\prime} \mathbf{1}\left(y-D<s_{i}^{\mathrm{E}}\right)\right] \\
& +\alpha \mathrm{E}\left[\left(G_{i}^{\mathrm{E}}(y-D)\right)^{\prime} \mathbf{1}\left(y-D \geq s_{i}^{\mathrm{E}}\right)\right] \\
\geq & -c_{i}^{\mathrm{R}}+c_{i}^{\mathrm{E}} P\left(y-D<s_{i}^{\mathrm{E}}\right)+\alpha c_{i}^{\mathrm{E}} P\left(y-D \geq s_{i}^{\mathrm{E}}\right) \\
& -\left(H_{1}+p-\sum_{j=1}^{i-1}\left(\alpha c_{j}^{\mathrm{E}}-c_{j}^{\mathrm{R}}\right)\right) \\
& \times P\left(D(i+1)>y, y-D<s_{i}^{\mathrm{E}}\right) \\
& -\alpha\left(H_{1}+p-\sum_{j=1}^{i-1}\left(\alpha c_{j}^{\mathrm{E}}-c_{j}^{\mathrm{R}}\right)\right) \\
& \times P\left(D(i+1)>y, y-D \geq s_{i}^{\mathrm{E}}\right) \\
\geq & -c_{i}^{\mathrm{R}}+\alpha c_{i}^{\mathrm{E}}-\left(H_{1}+p-\sum_{j=1}^{i-1}\left(\alpha c_{j}^{\mathrm{E}}-c_{j}^{\mathrm{R}}\right)\right) \\
& \times P(D(i+1)>y)
\end{aligned}
$$

where the first inequality follows from the fact that

$$
\left(G_{i}^{\mathrm{E}}(y)\right)^{\prime} \mathbf{1}\left(y \leq s_{i}^{\mathrm{E}}\right) \geq \mathrm{E}\left[\left(G_{i}^{\mathrm{E}}(y-D)\right)^{\prime} \mathbf{1}\left(y-D<s_{i}^{\mathrm{E}}\right)\right] .
$$

Therefore, if $\sum_{j=1}^{i}\left(\alpha c_{j}^{\mathrm{E}}-c_{j}^{\mathrm{R}}\right)>H_{1}+p,\left(G_{i}^{\mathrm{R}}(y)\right)^{\prime}>0$ and $s_{i}^{\mathrm{R}}=-\infty$. Moreover, as $\sum_{j=1}^{i+1}\left(\alpha c_{j}^{\mathrm{E}}-c_{j}^{\mathrm{R}}\right)=\alpha c_{i+1}^{\mathrm{E}}-c_{i+1}^{\mathrm{R}}+\sum_{j=1}^{i}\left(\alpha c_{j}^{\mathrm{E}}-c_{j}^{\mathrm{R}}\right)>H_{1}+p$, $s_{k}^{\mathrm{R}}=-\infty$ for $k \geq i$. And from the prior argument, $s_{k}^{\mathrm{E}}=-\infty$ for $k \geq i$.
Now, we prove Eq. (32) by induction. First, for $i=1$, Eq. (32) is equality. Suppose it is true for $i$. For $i+1$, note that

$$
\begin{gathered}
\left(G_{i+1}^{\mathrm{E}}(y)\right)^{\prime}=c_{i+1}^{\mathrm{E}}+\left(G_{i}^{\mathrm{R}}(y)\right)^{\prime} \mathbf{1}\left(y<s_{i}^{\mathrm{R}}\right) \\
\geq c_{i+1}^{\mathrm{E}}+\left[-c_{i}^{\mathrm{R}}+\alpha c_{i}^{\mathrm{E}}-\left(H_{1}+p-\sum_{j=1}^{i-1}\left(\alpha c_{j}^{\mathrm{E}}-c_{j}^{\mathrm{R}}\right)\right)\right. \\
\quad \times P(D(i+1)>y)] \mathbf{1}\left(y<s_{i}^{\mathrm{R}}\right) \\
\geq c_{i+1}^{\mathrm{E}}-\left(H_{1}+p-\sum_{j=1}^{i}\left(\alpha c_{j}^{\mathrm{E}}-c_{j}^{\mathrm{R}}\right)\right) P(D(i+1)>y)
\end{gathered}
$$

where the first inequality follows from the induction assumption and the second inequality follows from that $-c_{i}^{\mathrm{R}}+\alpha c_{i}^{\mathrm{E}} \geq 0$. So the proof is completed.

PROOF OF THEOREM 1: Since $s_{i}^{\mathrm{E}}$ and $s_{i}^{\mathrm{R}}$ are the solutions of $\left(G_{i}^{\mathrm{E}}(y)\right)^{\prime}=0$ and $\left(G_{i}^{\mathrm{R}}(y)\right)^{\prime}=0$, respectively, to prove the results, it is sufficient to show that for all $i$,

$$
\begin{aligned}
& \left(G_{i}^{\mathrm{E}}(y)\right)^{\prime} \leq \sum_{j=1}^{i}\left(c_{j}^{\mathrm{E}}-c_{j-1}^{\mathrm{R}}\right)-\left(H_{1}+p\right) P(D>y), \\
& \left(G_{i}^{\mathrm{R}}(y)\right)^{\prime} \leq-c_{i}^{\mathrm{R}}+\sum_{j=1}^{i}\left(c_{j}^{\mathrm{E}}-c_{j-1}^{\mathrm{R}}\right)-\left(H_{1}+p\right) P(D>y),
\end{aligned}
$$

and

$$
\begin{aligned}
& \left(G_{i}^{\mathrm{E}}(y)\right)^{\prime} \leq \sum_{j=1}^{i} \alpha^{i-j}\left(c_{j}^{\mathrm{E}}-c_{j-1}^{\mathrm{R}}\right)-\alpha^{i-1}\left(H_{1}+p\right) P(D>y) \\
& \left(G_{i}^{\mathrm{R}}(y)\right)^{\prime} \leq-c_{i}^{\mathrm{R}}+\sum_{j=1}^{i} \alpha^{i-j+1}\left(c_{j}^{\mathrm{E}}-c_{j-1}^{\mathrm{R}}\right)-\alpha^{i}\left(H_{1}+p\right) P(D>y)
\end{aligned}
$$

We prove these inequalities by induction. Consider Eqs. (34) and (35) first. Eq. (34) is clearly true for $s_{1}^{\mathrm{E}}$. We have shown Eq. (35) with $i=1$ in Section 3.

Assuming Eqs. (34) and (35) hold for $i$, we next prove that they hold for $i+1$. First, from Eqs. (1)-(4), an analogous idea as in the proof of $G_{2}^{\mathrm{E}}$ and the inductive assumption,

$$
\begin{aligned}
\left(G_{i+1}^{\mathrm{E}}(y)\right)^{\prime} & =c_{i+1}^{\mathrm{E}}+\left(G_{i}^{\mathrm{R}}\left(y \wedge s_{i}^{\mathrm{R}}\right)\right)^{\prime} \\
& \leq c_{i+1}^{\mathrm{E}}+\left(G_{i}^{\mathrm{R}}(y)\right)^{\prime} \\
& \leq c_{i+1}^{\mathrm{E}}-c_{i}^{\mathrm{R}}+\sum_{j=1}^{i}\left(c_{j}^{\mathrm{E}}-c_{j-1}^{\mathrm{R}}\right)-\left(H_{1}+p\right) P(D>y) \\
& =\sum_{j=1}^{i+1}\left(c_{j}^{\mathrm{E}}-c_{j-1}^{\mathrm{R}}\right)-\left(H_{1}+p\right) P(D>y) .
\end{aligned}
$$

We then prove Eq. (35) for $i+1$. Note that

$$
\begin{aligned}
\left(G_{i+1}^{\mathrm{R}}(y)\right)^{\prime}= & -c_{i+1}^{\mathrm{R}}+\left(G_{i+1}^{\mathrm{E}}\right)^{\prime}(y) \mathbf{1}\left(y<s_{i+1}^{\mathrm{E}}\right) \\
& +\alpha \mathrm{E}\left[\left(G_{i+1}^{\mathrm{E}}\right)^{\prime}(y-D) \mathbf{1}\left(y-D \geq s_{i+1}^{\mathrm{E}}\right)\right] \\
\leq & -c_{i+1}^{\mathrm{R}}+\mathrm{E}\left[\left(G_{i+1}^{\mathrm{E}}\right)^{\prime}(y) \mathbf{1}\left(y<s_{i+1}^{\mathrm{E}}\right)\right] \\
& +\left(G_{i+1}^{\mathrm{E}}\right)^{\prime}(y) \mathbf{1}\left(y \geq s_{i+1}^{\mathrm{E}}\right) \\
\leq & -c_{i+1}^{\mathrm{R}}+\sum_{j=1}^{i+1}\left(c_{j}^{\mathrm{E}}-c_{j-1}^{\mathrm{R}}\right)-\left(H_{1}+p\right) P(D>y),
\end{aligned}
$$


where the first inequality follows from the convexity of $G_{i+1}^{\mathrm{E}}(y)$ and $0 \leq \alpha<1$ and the second inequality is from the inductive assumption.

We proceed to show Eqs. (36) and (37). The inequality Eq. (36) is clearly true for $i=1$. For Eq. (37) and $i=1$,

$$
\begin{aligned}
\left(G_{1}^{\mathrm{R}}(y)\right)^{\prime} & =-c_{1}^{\mathrm{R}}+\left(G_{1}^{\mathrm{E}}(y)\right)^{\prime} \mathbf{1}\left(y<s_{1}^{\mathrm{E}}\right)+\alpha \mathrm{E}\left[\left(G_{1}^{\mathrm{E}}\right)^{\prime}\left((y-D) \vee s_{1}^{\mathrm{E}}\right)\right] \\
& \leq-c_{1}^{\mathrm{R}}+\alpha\left(G_{1}^{\mathrm{E}}(y)\right)^{\prime} \mathbf{1}\left(y<s_{1}^{\mathrm{E}}\right)+\alpha\left(G_{1}^{\mathrm{E}}\right)^{\prime}\left(y \vee s_{1}^{\mathrm{E}}\right) \\
& =-c_{1}^{\mathrm{R}}+\alpha c_{1}^{\mathrm{E}}-\alpha\left(H_{1}+p\right) P(D>y),
\end{aligned}
$$

where the inequality follows from the convexity of $G_{1}^{\mathrm{E}}$.

Assuming Eqs. (36) and (37) hold for $i$, we prove that they hold for $i+1$. First, for Eq. (36),

$$
\begin{aligned}
\left(G_{i+1}^{\mathrm{E}}(y)\right)^{\prime} \leq & c_{i+1}^{\mathrm{E}}+\left(G_{i}^{\mathrm{R}}(y)\right)^{\prime} \\
\leq & c_{i+1}^{\mathrm{E}}-c_{i}^{\mathrm{R}}+\sum_{j=1}^{i} \alpha^{i-j+1}\left(c_{j}^{\mathrm{E}}-c_{j-1}^{\mathrm{R}}\right) \\
& -\alpha^{i}\left(H_{1}+p\right) P(D>y) \\
= & \sum_{j=1}^{i+1} \alpha^{i-j+1}\left(c_{j}^{\mathrm{E}}-c_{j-1}^{\mathrm{R}}\right)-\alpha^{i}\left(H_{1}+p\right) P(D>y),
\end{aligned}
$$

where the second inequality follows from the inductive assumption of Eq. (37).

We finally prove Eq. (37) for $i+1$. Note that

$$
\begin{aligned}
\left(G_{i+1}^{\mathrm{R}}(y)\right)^{\prime}= & -c_{i+1}^{\mathrm{R}}+\left(G_{i+1}^{\mathrm{E}}\right)^{\prime}(y) \mathbf{1}\left(y<s_{i+1}^{\mathrm{E}}\right) \\
& +\alpha \mathrm{E}\left[\left(G_{i+1}^{\mathrm{E}}\right)^{\prime}(y-D) \mathbf{1}\left(y-D \geq s_{i+1}^{\mathrm{E}}\right)\right] \\
\leq & -c_{i+1}^{\mathrm{R}}+\alpha\left(G_{i+1}^{\mathrm{E}}\right)^{\prime}(y) \mathbf{1}\left(y<s_{i+1}^{\mathrm{E}}\right) \\
& +\alpha \mathrm{E}\left[\left(G_{i+1}^{\mathrm{E}}\right)^{\prime}(y-D) \mathbf{1}\left(y-D \geq s_{i+1}^{\mathrm{E}}\right)\right] \\
\leq & -c_{i+1}^{\mathrm{R}}+\alpha\left(G_{i+1}^{\mathrm{E}}\right)^{\prime}(y) \mathbf{1}\left(y<s_{i+1}^{\mathrm{E}}\right) \\
& +\alpha\left(G_{i+1}^{\mathrm{E}}\right)^{\prime}(y) \mathbf{1}\left(y \geq s_{i+1}^{\mathrm{E}}\right) \\
\leq & -c_{i+1}^{\mathrm{R}}+\left(\sum_{j=1}^{i+1} \alpha^{i-j+2}\left(c_{j}^{\mathrm{E}}-c_{j-1}^{\mathrm{R}}\right)\right) \\
& -\alpha^{i+1}\left(H_{1}+p\right) P(D>y),
\end{aligned}
$$

where the inequalities again follow from the convexity of $G_{i+1}^{\mathrm{E}}$ and $0 \leq \alpha<1$. Hence, the proof is completed.

PROOF OF THEOREM 2: For Eqs. (17) and (18), we need to show for $i=2, \ldots, N$,

$$
\begin{array}{r}
\left(G_{i}^{\mathrm{E}}(y)\right)^{\prime} \leq-A_{i, i-k+1}+\sum_{j=1}^{i-k+1} \alpha^{i-j}\left(c_{j}^{\mathrm{E}}-c_{j-1}^{\mathrm{R}}\right) P(D(k) \leq y), \\
k=2, \ldots, i,
\end{array}
$$

and $i=1, \ldots, N$,

$$
\begin{aligned}
& \left(G_{i}^{\mathrm{R}}(y)\right)^{\prime} \leq-B_{i, i-k+1} \\
& \quad+\sum_{j=1}^{i-k+1} \alpha^{i-j+1}\left(c_{j}^{\mathrm{E}}-c_{j-1}^{\mathrm{R}}\right) P(D(k+1) \leq y), \quad k=1, \ldots, i .
\end{aligned}
$$

For $i=1, \ldots, N$, from Theorem 1 ,

$$
\begin{aligned}
\left(G_{i}^{\mathrm{R}}(y)\right)^{\prime}= & -c_{i}^{\mathrm{R}}+\left(G_{i}^{\mathrm{E}}(y)\right)^{\prime} \mathbf{1}\left(y<s_{i}^{\mathrm{E}}\right) \\
& +\alpha \mathrm{E}\left[\left(G_{i}^{\mathrm{E}}\right)^{\prime}(y-D) \mathbf{1}\left(y-D \geq s_{i}^{\mathrm{E}}\right)\right] \\
\leq & -c_{i}^{\mathrm{R}}+\alpha \mathrm{E}\left[\left(G_{i}^{\mathrm{E}}\right)^{\prime}(y-D) \mathbf{1}\left(y-D \geq s_{i}^{\mathrm{E}}\right)\right] \\
\leq & -c_{i}^{\mathrm{R}}+\alpha\left[\sum_{j=1}^{i} \alpha^{i-j}\left(c_{j}^{\mathrm{E}}-c_{j-1}^{\mathrm{R}}\right) P\left(y-D \geq s_{i}^{\mathrm{E}}\right)\right. \\
& \left.-\alpha^{i-1}\left(H_{1}+p\right) P\left(D(2)>y, y-D \geq s_{i}^{\mathrm{E}}\right)\right] \\
\leq & -c_{i}^{\mathrm{R}}+\alpha\left[\sum_{j=1}^{i} \alpha^{i-j}\left(c_{j}^{\mathrm{E}}-c_{j-1}^{\mathrm{R}}\right) P\left(y-D \geq s_{i}^{\mathrm{E}}\right)\right. \\
& \left.-\sum_{j=1}^{i} \alpha^{i-j}\left(c_{j}^{\mathrm{E}}-c_{j-1}^{\mathrm{R}}\right) P\left(D(2)>y, y-D \geq s_{i}^{\mathrm{E}}\right)\right] \\
= & -c_{i}^{\mathrm{R}}+\alpha\left[\sum_{j=1}^{i} \alpha^{i-j}\left(c_{j}^{\mathrm{E}}-c_{j-1}^{\mathrm{R}}\right) P\left(y-D \geq s_{i}^{\mathrm{E}}, D(2) \leq y\right)\right] \\
\leq & -c_{i}^{\mathrm{R}}+\sum_{j=1}^{i} \alpha^{i-j+1}\left(c_{j}^{\mathrm{E}}-c_{j-1}^{\mathrm{R}}\right) P(D(2) \leq y) \\
= & -B_{i, i}+\sum_{j=1}^{i} \alpha^{i-j+1}\left(c_{j}^{\mathrm{E}}-c_{j-1}^{\mathrm{R}}\right) P(D(2) \leq y),
\end{aligned}
$$

where the third inequality follows from the assumption that $\sum_{j=1}^{i} \alpha^{i-j}\left(c_{j}^{\mathrm{E}}-\right.$ $\left.c_{j-1}^{\mathrm{R}}\right) \leq \alpha^{i-1}\left(H_{1}+p\right)$. This validates the case $k=1$ for Eq. 39. The derivation above implies that

$$
\begin{aligned}
\left(G_{i+1}^{\mathrm{E}}(y)\right)^{\prime} & =c_{i+1}^{\mathrm{E}}+\left(G_{i}^{\mathrm{R}}(y)\right)^{\prime} \mathbf{1}\left(y<s_{i}^{\mathrm{R}}\right) \\
& \leq c_{i+1}^{\mathrm{E}}+\left(G_{i}^{\mathrm{R}}(y)\right)^{\prime} \\
& \leq c_{i+1}^{\mathrm{E}}-c_{i}^{\mathrm{R}}+\sum_{j=1}^{i} \alpha^{i-j+1}\left(c_{j}^{\mathrm{E}}-c_{j-1}^{\mathrm{R}}\right) P(D(2) \leq y) \\
& \leq-A_{i+1, i}+\sum_{j=1}^{i} \alpha^{i-j+1}\left(c_{j}^{\mathrm{E}}-c_{j-1}^{\mathrm{R}}\right) P(D(2) \leq y) .
\end{aligned}
$$

This proves the case for $k=2$ of Eq. (38).

On the basis of these, suppose Eq. (38) holds for some $k=j$, then for Eq. (39),

$$
\begin{aligned}
\left(G_{i}^{\mathrm{R}}(y)\right)^{\prime} \leq & -c_{i}^{\mathrm{R}}+\alpha \mathrm{E}\left[\left(G_{i}^{\mathrm{E}}\right)^{\prime}(y-D) \mathbf{1}\left(y-D \geq s_{i}^{\mathrm{E}}\right)\right] \\
\leq & -c_{i}^{\mathrm{R}}+\alpha \mathrm{E}\left[-A_{i, i-j+1} P\left(y-D \geq s_{i}^{\mathrm{E}}\right)\right. \\
& \left.+\sum_{l=1}^{i-j+1} \alpha^{i-l}\left(c_{l}^{\mathrm{E}}-c_{l-1}^{\mathrm{R}}\right) P\left(D(j+1) \leq y, y-D \geq s_{i}^{\mathrm{E}}\right)\right] \\
\leq & -B_{i, i-j+1}+\sum_{l=1}^{i-j+1} \alpha^{i-l+1}\left(c_{l}^{\mathrm{E}}-c_{l-1}^{\mathrm{R}}\right) P(D(j+1) \leq y),
\end{aligned}
$$

Naval Research Logistics DOI 10.1002/nav 
thus Eq. (39) holds for $k=j+1$. Meanwhile,

$$
\begin{aligned}
\left(G_{i+1}^{\mathrm{E}}(y)\right)^{\prime}= & c_{i+1}^{\mathrm{E}}+\left(G_{i}^{\mathrm{R}}(y)\right)^{\prime} \mathbf{1}\left(y<s_{i}^{\mathrm{R}}\right) \\
\leq & c_{i+1}^{\mathrm{E}}+\left(G_{i}^{\mathrm{R}}(y)\right)^{\prime} \\
\leq & c_{i+1}^{\mathrm{E}}-c_{i}^{\mathrm{R}}+\alpha A_{i, i-j+1}^{-} \\
& +\sum_{l=1}^{i} \alpha^{i-l+1}\left(c_{l}^{\mathrm{E}}-c_{l-1}^{\mathrm{R}}\right) P(D(j+1) \leq y) \\
= & -A_{i+1, i-j+1}+\sum_{l=1}^{i-j+1} \alpha^{i-l+1}\left(c_{l}^{\mathrm{E}}-c_{l-1}^{\mathrm{R}}\right) P(D(j+1) \leq y),
\end{aligned}
$$

which verifies that Eq. (38) holds for $k=j+1$. So, we complete the induction proof.

PROOF OF THEOREM 3: It suffices to show that for $i=1,2, \ldots, N$,

$$
\left(G_{i}^{\mathrm{E}}(y)\right)^{\prime} \leq c_{i}^{\mathrm{E}}-c_{i-1}^{\mathrm{R}}+\alpha c_{i-1}^{\mathrm{E}} P\left(D \leq y-s_{i-1}^{\mathrm{E}}\right),
$$

and

$$
\left(G_{i}^{\mathrm{R}}(y)\right)^{\prime} \leq-c_{i}^{\mathrm{R}}+\alpha c_{i}^{\mathrm{E}} P\left(D \leq y-s_{i}^{\mathrm{E}}\right) .
$$

For Eq. (40), note that

$$
\begin{aligned}
\left(G_{i}^{\mathrm{E}}(y)\right)^{\prime} & =c_{i}^{\mathrm{E}}+\left(G_{i-1}^{\mathrm{R}}(y)\right)^{\prime} \mathbf{1}\left(y<s_{i-1}^{\mathrm{R}}\right) \\
& \leq c_{i}^{\mathrm{E}}+\left(G_{i-1}^{\mathrm{R}}(y)\right)^{\prime} \\
& \leq c_{i}^{\mathrm{E}}-c_{i-1}^{\mathrm{R}}+\alpha \mathrm{E}\left[\left(G_{i-1}^{\mathrm{E}}(y-D)\right)^{\prime} \mathbf{1}\left(y-D \geq s_{i-1}^{\mathrm{E}}\right)\right] \\
& \leq c_{i}^{\mathrm{E}}-c_{i-1}^{\mathrm{R}}+\alpha c_{i-1}^{\mathrm{E}} P\left(y-D \geq s_{i-1}^{\mathrm{E}}\right)
\end{aligned}
$$

where the last inequality follows from that $\left(G_{i-1}^{\mathrm{E}}(y)\right)^{\prime} \leq c_{i-1}^{\mathrm{E}}$. The inequality Eq. (41) follows from

$$
\begin{aligned}
\left(G_{i}^{\mathrm{R}}(y)\right)^{\prime} & \leq-c_{i}^{\mathrm{R}}+\alpha \mathrm{E}\left[\left(G_{i}^{\mathrm{E}}(y-D)\right)^{\prime} \mathbf{1}\left(y-D \geq s_{i}^{\mathrm{E}}\right)\right] \\
& \leq-c_{i}^{\mathrm{R}}+\alpha c_{i}^{\mathrm{E}} P\left(y-D \geq s_{i}^{\mathrm{E}}\right) .
\end{aligned}
$$

Therefore, the theorem is proved.

PROOF OF THEOREM 4: Recall the inequality Eq. (32). As $s_{i}^{\mathrm{E}} \leq s_{i-1}^{\mathrm{R}}$, the solution of

$c_{i}^{\mathrm{E}}+\left[-c_{i-1}^{\mathrm{R}}+\alpha c_{i-1}^{\mathrm{E}}-\left(H_{1}+p-\sum_{j=1}^{i-2}\left(\alpha c_{j}^{\mathrm{E}}-c_{j}^{\mathrm{R}}\right)\right) P(D(i)>y)\right]=0$ must be an upper bound of $s_{i}^{\mathrm{E}}$, i.e.,

$$
\bar{s}_{i}^{\mathrm{E} 1}=\bar{F}_{i}^{-1}\left(\frac{c_{i}^{\mathrm{E}}-c_{i-1}^{\mathrm{R}}+\alpha c_{i-1}^{\mathrm{E}}}{H_{1}+p-\sum_{j=1}^{i-2}\left(\alpha c_{j}^{\mathrm{E}}-c_{j}^{\mathrm{R}}\right)}\right) \geq s_{i}^{\mathrm{E}} .
$$

To see this, note that if $\bar{s}_{i}^{E 1}<s_{i-1}^{\mathrm{R}}$ then by Eq. (33), we have on $y \leq s_{i-1}^{\mathrm{R}}$,

$$
\begin{aligned}
&\left(G_{i}^{\mathrm{E}}(y)\right)^{\prime} \geq c_{i}^{\mathrm{E}} \\
& \quad+\left[-c_{i-1}^{\mathrm{R}}+\alpha c_{i-1}^{\mathrm{E}}-\left(H_{1}+p-\sum_{j=1}^{i-2}\left(\alpha c_{j}^{\mathrm{E}}-c_{j}^{\mathrm{R}}\right)\right) P(D(i)>y)\right],
\end{aligned}
$$

hence, $\left(G_{i}^{\mathrm{E}}\left(\bar{s}_{i}^{E 1}\right)\right)^{\prime} \geq 0$, and it implies $\bar{s}_{i}^{E 1} \geq s_{i}^{\mathrm{E}}$; On the other hand, if $\bar{s}_{i}^{E 1} \geq s_{i-1}^{\mathrm{R}}$, then the result holds automatically because $s_{i}^{\mathrm{E}} \leq s_{i-1}^{\mathrm{R}}$.

We next show Eq. (22). Note that $\left(G_{i}^{\mathrm{E}}(y)\right)^{\prime} 1\left(y \leq s_{i}^{\mathrm{E}}\right)=0$ on $y \geq s_{i}^{\mathrm{E}}$. Hence, as $s_{i}^{\mathrm{R}} \geq s_{i}^{\mathrm{E}}$ and for $y \geq s_{i}^{\mathrm{E}}$, from Eq. (32),

$$
\begin{aligned}
& \left(G_{i}^{\mathrm{R}}(y)\right)^{\prime}=-c_{i}^{\mathrm{R}}+\alpha \mathrm{E}\left[\left(G_{i}^{\mathrm{E}}(y-D)\right)^{\prime} \mathbf{1}\left(y-D \geq s_{i}^{\mathrm{E}}\right)\right] \\
& \quad \geq-c_{i}^{\mathrm{R}}+\alpha c_{i}^{\mathrm{E}}-\alpha\left(H_{1}+p-\sum_{j=1}^{i-1}\left(\alpha c_{j}^{\mathrm{E}}-c_{j}^{\mathrm{R}}\right)\right) P(D(i+1)>y),
\end{aligned}
$$

which implies Eq. (22). To verify Eq. (22) is indeed an upper bound for $s_{i}^{\mathrm{R}}$, we still need to show it is greater than or equal to $s_{i}^{\mathrm{E}}$. This is true, since plugging $y=s_{i}^{\mathrm{E}}$ in Eq. (42) shows that the right hand side is negative, implying that Eq. (22) is at least as large as $s_{i}^{\mathrm{E}}$.

PROOF OF THEOREM 5: The validity of Eq. (24) follows from Proposition 1. For Eq. (25), note that

$$
\begin{aligned}
& \left(\mathrm{E}\left[G_{i}^{\mathrm{E}}(y-D)\right]\right)^{\prime}=c_{i}^{\mathrm{E}}+\mathrm{E}\left[\left(G_{i-1}^{\mathrm{R}}(y-D)\right)^{\prime} \mathbf{1}\left(y-D<s_{i-1}^{\mathrm{R}}\right)\right] \\
& \geq c_{i}^{\mathrm{E}}+\mathrm{E}\left[\left(-c_{i-1}^{\mathrm{R}}+\alpha c_{i-1}^{\mathrm{E}}-\left(H_{1}+p-\sum_{j=1}^{i-2}\left(\alpha c_{j}^{\mathrm{E}}-c_{j}^{\mathrm{R}}\right)\right) \mathbf{1}(D(i)>y)\right)\right. \\
& \left.\times \mathbf{1}\left(y-D<s_{i-1}^{\mathrm{R}}\right)\right] \\
& \geq c_{i}^{\mathrm{E}}+\mathrm{E}\left[\left(-\left(H_{1}+p-\sum_{j=1}^{i-1}\left(\alpha c_{j}^{\mathrm{E}}-c_{j}^{\mathrm{R}}\right)\right) \mathbf{1}(D(i)>y)\right)\right. \\
& \left.\times \mathbf{1}\left(y-D<s_{i-1}^{\mathrm{R}}\right)\right] \\
& \geq c_{i}^{\mathrm{E}}-\left(H_{1}+p-\sum_{j=1}^{i-1}\left(\alpha c_{j}^{\mathrm{E}}-c_{j}^{\mathrm{R}}\right)\right) P\left(D>y-s_{i-1}^{\mathrm{R}}\right),
\end{aligned}
$$

where the first inequality follows from Eq. (42), the second one follows from $-c_{i-1}^{\mathrm{R}}+\alpha c_{i-1}^{\mathrm{E}} \geq 0$ and the last one from $H_{1}+p-\sum_{j=1}^{i-1}\left(\alpha c_{j}^{\mathrm{E}}-c_{j}^{\mathrm{R}}\right) \geq 0$. Moreover, as $s_{i}^{\mathrm{R}} \geq s_{i}^{\mathrm{E}}$ and for $y \geq s_{i}^{\mathrm{E}}$,

$$
\begin{aligned}
\left(G_{i}^{\mathrm{R}}(y)\right)^{\prime} & =-c_{i}^{\mathrm{R}}+\alpha \mathrm{E}\left[\left(G_{i}^{\mathrm{E}}(y-D)\right)^{\prime} \mathbf{1}\left(y-D \geq s_{i}^{\mathrm{E}}\right)\right] \\
& \geq-c_{i}^{\mathrm{R}}+\alpha \mathrm{E}\left[\left(G_{i}^{\mathrm{E}}(y-D)\right)^{\prime}\right] \\
& \geq-c_{i}^{\mathrm{R}}+\alpha c_{i}^{\mathrm{E}}-\alpha\left(H_{1}+p-\sum_{j=1}^{i-1}\left(\alpha c_{j}^{\mathrm{E}}-c_{j}^{\mathrm{R}}\right)\right) P\left(D>y-s_{i-1}^{\mathrm{R}}\right),
\end{aligned}
$$

which implies the second term in the brackets of Eq. (25). It can be shown that the solution of Eq. (43) is greater than or equal to $s_{i}^{\mathrm{E}}$ since plugging $y=s_{i}^{\mathrm{E}}$ in the equations above shows that the right hand side of Eq. (43) is negative. For the first term in Eq. (25), note that for $y>s_{i}^{\mathrm{E}}$,

$$
\begin{aligned}
\left(G_{i}^{\mathrm{R}}(y)\right)^{\prime} & =-c_{i}^{\mathrm{R}}+\alpha \mathrm{E}\left[\left(G_{i}^{\mathrm{E}}(y-D)\right)^{\prime} 1\left(y-D \geq s_{i}^{\mathrm{E}}\right)\right] \\
& \geq-c_{i}^{\mathrm{R}}+\alpha \mathrm{E}\left[\left(G_{i}^{\mathrm{E}}(y-D)\right)^{\prime} 1\left(y-D \geq s_{i-1}^{\mathrm{R}}\right)\right] \\
& \geq-c_{i}^{\mathrm{R}}+\alpha c_{i}^{\mathrm{E}} P\left(y-D \geq s_{i-1}^{\mathrm{R}}\right) \\
& =-c_{i}^{\mathrm{R}}+\alpha c_{i}^{\mathrm{E}}-\alpha c_{i}^{\mathrm{E}} P\left(y-D<s_{i-1}^{\mathrm{R}}\right),
\end{aligned}
$$

where the first inequality follows from that $\left(G_{i}^{\mathrm{E}}(y)\right)^{\prime} \leq 0$ for $y \leq s_{i}^{\mathrm{E}}$ and $s_{i}^{\mathrm{E}} \leq s_{i-1}^{\mathrm{R}}$ and the second inequality follows from the definition of $G_{i}^{\mathrm{E}}(y)$ for $y>s_{i-1}^{\mathrm{R}}$. Therefore, Eq. (25) is valid.

Naval Research Logistics DOI 10.1002/nav 
PROOF OF THEOREM 6: We first show that, for $i=1, \ldots, N$,

$$
\left(G_{i}^{\mathrm{E}}(y)\right)^{\prime} \geq C_{i}-\left(H_{1}+p\right) P(D>y) .
$$

This implies the first part of Eq. (26).

As $s_{i}^{\mathrm{R}} \geq s_{i}^{\mathrm{E}}$, we have on $y \geq s_{i}^{\mathrm{E}}$,

$$
\begin{aligned}
\left(G_{i}^{\mathrm{R}}(y)\right)^{\prime}= & -c_{i}^{\mathrm{R}}+\left(G_{i}^{\mathrm{E}}(y)\right)^{\prime} \mathbf{1}\left(y \leq s_{i}^{\mathrm{E}}\right) \\
& +\alpha \mathrm{E}\left[\left(G_{i}^{\mathrm{E}}(y-D)\right)^{\prime} \mathbf{1}\left(y-D \geq s_{i}^{\mathrm{E}}\right)\right] \\
= & -c_{i}^{\mathrm{R}}+\alpha \mathrm{E}\left[\left(G_{i}^{\mathrm{E}}(y-D)\right)^{\prime} \mathbf{1}\left(y-D \geq s_{i}^{\mathrm{E}}\right)\right] \\
\geq & -c_{i}^{\mathrm{R}}+\alpha \mathrm{E}\left[\left(G_{i}^{\mathrm{E}}(y-D)\right)^{\prime}\right] \\
\geq & -c_{i}^{\mathrm{R}}+\alpha C_{i}-\alpha\left(H_{1}+p\right) P(D(2)>y),
\end{aligned}
$$

which implies Eq. (27). To show that Eq. (27) is greater than or equal to $s_{i}^{\mathrm{E}}$, plugging $y=s_{i}^{\mathrm{E}}$ in the equation above shows that the right hand side is negative and we obtain the desired result.

We prove Eq. (44) by induction. The case of $i=1$ is similar to that of Theorem 3 so we skip it here. Suppose it is true for $i$, and we proceed to prove $i+1$.

$$
\begin{aligned}
\left(G_{i+1}^{\mathrm{E}}(y)\right)^{\prime}= & c_{i+1}^{\mathrm{E}}+\left(G_{i}^{\mathrm{R}}(y)\right)^{\prime} \mathbf{1}\left(y \leq s_{i}^{\mathrm{R}}\right) \\
= & c_{i+1}^{\mathrm{E}}+\left(G_{i}^{\mathrm{E}}(y)\right)^{\prime} \mathbf{1}\left(y \leq s_{i}^{\mathrm{E}}\right) \mathbf{1}\left(y \leq s_{i}^{\mathrm{R}}\right) \\
& +\left(-c_{i}^{\mathrm{R}}+\alpha \mathrm{E}\left[\left(G_{i}^{\mathrm{E}}(y-D)\right)^{\prime} \mathbf{1}\left(y-D \geq s_{i}^{\mathrm{E}}\right)\right]\right) \mathbf{1}\left(y \leq s_{i}^{\mathrm{R}}\right) \\
\geq & c_{i+1}^{\mathrm{E}}+\left(C_{i}-\left(H_{1}+p\right) P(D>y)\right) \mathbf{1}\left(y \leq s_{i}^{\mathrm{E}}\right)-c_{i}^{\mathrm{R}} \\
\geq & c_{i+1}^{\mathrm{E}}-c_{i}^{\mathrm{R}}-C_{i}^{-}-\left(H_{1}+p\right) P(D>y) \\
= & C_{i+1}-\left(H_{1}+p\right) P(D>y)
\end{aligned}
$$

where the first inequality follows from the inductive assumption and $\left(G_{i}^{\mathrm{E}}(y-D)\right)^{\prime} \mathbf{1}\left(y-D \geq s_{i}^{\mathrm{E}}\right) \geq 0$. Moreover, as $s_{i+1}^{\mathrm{E}} \leq s_{i}^{\mathrm{R}}$, we can obtain another upper bound which is the solution of

$$
c_{i+1}^{\mathrm{E}}-c_{i}^{\mathrm{R}}+\min \left\{\alpha C_{i}, C_{i}\right\}-\left(H_{1}+p\right) P(D(2)>y)=0 .
$$

This implies the second term in the brackets of Eq. (26). Thus, we complete the proof.

\section{ACKNOWLEDGMENTS}

The authors thank the Associate Editor and two anonymous referees for their helpful comments and suggestions, which have helped greatly to improve the exposition of this article. This work was partially supported by Hong Kong GRF grant CUHK 419408 for the first author, and NSF grants CMMI-0800004 and CMMI-0927631 for the second author.

\section{REFERENCES}

[1] E.W. Barankin, A delivery-lag inventory model with an emergency provision (the one-period case), Naval Res Logist Quart 8 (1961), 258-311.
[2] X. Chao and S.X. Zhou, Probabilistic solution and bounds for serial systems with discounted and average costs, Naval Res Logist 54 (2007), 623-631.

[3] F. Chen and Y-S. Zheng, Lower bounds for multi-echelon stochastic inventory systems, Manage Sci 40 (1994), 1426-1443.

[4] A. Clark and H. Scarf, Optimal policies for the multi-echelon inventory problem, Manage Sci 6 (1960), 475-490.

[5] K. Daniel, "A delivery-lag inventory model with emergency," Multistage inventory models and techniques, H. Scarf, D. Giligord, and M. Sheely (Editors), Stanford University Press, Stanford, CA, 1963.

[6] L.X. Dong and H.L. Lee, Optimal policies and approximations for a serial multiechelon inventory system with time-correlated demand, Oper Res 51 (2003), 969-980.

[7] A. Federgruen and P. Zipkin, Computational issues in an infinite horizon multi-echelon inventory problem with stochastic demand, Oper Res 32 (1984), 818-836.

[8] Q. Feng, G. Gallego, S.P. Sethi, H. Yan, and H. Zhang, "A periodic review inventory model with three consecutive delivery modes and forecast updates," Working Paper, University of Texas at Dallas, Richardson, TX, 2003.

[9] Q. Feng, G. Gallego, S.P. Sethi, H. Yan, and H. Zhang, "Optimality and nonoptimality of the base-stock policy in inventory problems with multiple delivery modes," Working Paper, University of Texas at Dallas, Richardson, TX, 2004.

[10] Y. Fukuda, Optimal policies for the inventory problem with negotiable leadtime, Manage Sci 10 (1964), 690-708.

[11] G. Gallego and P.H. Zipkin, Stock positioning and performance estimation in serial production-transportation system, Manuf Serv Oper Manage 1 (1999), 77-88.

[12] G. Gallego and O. Özer, A new algorithm and a new heuristic for serial supply systems, Oper Res Lett 33 (2006), 349-362.

[13] D.G. Lawson and E.L. Porteus, Multistage inventory management with expediting, Oper Res 48 (2000), 878-893.

[14] A. Muharremoglu and J. Tsitsiklis, "Dynamic leadtime management in supply chains," Working paper, Columbia University, New York, NY, 2003.

[15] A. Scheller-Wolf, G.J. van Houtum, and S. Veeraraghavan, "Inventory models with expedited ordering: Single index policies (Unabridged Version)," Working Paper, Carnegie Mellon University, Pittsburgh, PA, 2003.

[16] K.H. Shang and J.S. Song, Newsvendor bounds and heuristics for optimal policies in serial supply chains, Manage Sci 49 (2003), 618-638.

[17] A. Sheopuri, G. Janakiraman, and S. Seshadri, "New policies for the stochastic inventory control problem with two supply sources," Working Paper, Stern School of Business, New York University, NY, 2007.

[18] J.S. Song and P. Zipkin, Inventories with multiple supply sources and networks of queues with overflow bypasses, Manage Sci 55 (2009), 362-372.

[19] S. Veeraraghavan and A. Scheller-Wolf, Now or later: A simple policy for effective dual sourcing in capacitated systems, Oper Res 56 (2008), 850-864.

[20] A.S. Whittemore and S.C. Saunders, Optimal inventory under stochastic demand with two supply options, SIAM J Appl Math 32 (1977), 293-305.

[21] P. Zipkin, Foundations of inventory management, McGrawHill Press, New York, 2000. 